\title{
Partition-Matrix Theory Applied to the Computation of Generalized-Inverses for MIMO Systems in Rayleigh Fading Channels
}

\author{
P. Cervantes1, L. F. González1, F. J. Ortiz2 and
}

A. D. García2

Additional information is available at the end of the chapter

http://dx.doi.org/10.5772/48198

\section{Introduction}

Partition-Matrix Theory and Generalized-Inverses are interesting topics explored in linear algebra and matrix computation. Partition-Matrix Theory is associated with the problem of properly partitioning a matrix into block matrices (i.e. an array of matrices), and is a matrix computation tool widely employed in several scientific-technological application areas. For instance, blockwise Toeplitz-based covariance matrices are used to model structural properties for space-time multivariate adaptive processing in radar applications [1], Jacobian response matrices are partitioned into several block-matrix instances in order to enhance medical images for Electrical-Impedance-Tomography [2], design of state-regulators and partial-observers for non-controllable/non-observable linear continuous systems contemplates matrix blocks for controllable/non-controllable and observable/non-observable eigenvalues [3]. The Generalized-Inverse is a common and natural problem found in a vast of applications. In control robotics, non-collocated partial linearization is applied to underactuated mechanical systems through inertia-decoupling regulators which employ a pseudoinverse as part of a modified input control law [4]. At sliding-mode control structures, a RightPseudoinverse is incorporated into a state-feedback control law in order to stabilize electromechanical non-linear systems [5]. Under the topic of system identification, definition of a Left-Pseudoinverse is present in auto-regressive moving-average models (ARMA) for matching dynamical properties of unknown systems [6]. An interesting approach arises whenever Partition-Matrix Theory and Generalized-Inverse are combined together yielding attractive solutions for solving the problem of block matrix inversion [7-10]. Nevertheless, 
several assumptions and restrictions regarding numerical stability and structural properties are considered for these alternatives. For example, an attractive pivot-free block matrix inversion algorithm is proposed in [7], which unfortunately exhibits an overhead in matrix multiplications that are required in order to guarantee full-rank properties for particular blocks within it. For circumventing the expense in rank deficiency, [8] offers block-matrix completion strategies in order to find the Generalized-Inverse of any non-singular block matrix (irrespective of the singularity of their constituting sub-blocks). However, the existence of intermediate matrix inverses and pseudoinverses throughout this algorithm still rely on full-rank assumptions, as well as introducing more hardness to the problem. The proposals exposed in [9-10] avoid completion strategies and contemplate all possible scenarios for avoiding any rank deficiency among each matrix sub-block, yet demanding full-rank assumptions for each scenario. In this chapter, an iterative-recursive algorithm for computing a Left-Pseudoinverse (LPI) of a MIMO channel matrix is developed by combining PartitionMatrix Theory and Generalized-Inverse concepts. For this approach, no matrix-operations' overhead nor any particular block matrix full-rank assumptions are needed because of structural attributes of the MIMO channel matrix, which models dynamical properties of a Rayleigh fading channel (RFC) within wireless MIMO communication systems.

The content of this work is outlined as follows. Section 2 provides a description of the MIMO communication link, pointing out its principal physical effects and the mathematical model considered for RFC-based environments. Section 3 defines formally the problem of computing the Left-Pseudoinverse as the Generalized-Inverse for the MIMO channel matrix applying Partition-Matrix Theory concepts. Section 4 presents linear algebra and matrix computation concepts and tools needed for tracking a solution for the aforementioned problem. Section 5 analyzes important properties of the MIMO channel matrix derived from a Rayleigh fading channel scenario. Section 6 explains the proposed novel algorithm. Section 7 presents a brief analysis of VLSI (Very Large Scale of Integration) aspects towards implementation of arithmetic operations presented in this algorithm. Section 8 concludes the chapter. Due to the vast literature about MIMO systems, and to the best of the authors' knowledge, this chapter provides a nice and strategic list of references in order to easily correlate essential concepts between matrix theory and MIMO systems. For instance, [11-16] describe and analyze information and system aspects about MIMO communication systems, as well as studying MIMO channel matrix behavior under RFC-based environments; [17-18] contain all useful linear algebra and matrix computation theoretical concepts around the mathematical background immersed in MIMO systems; [19-21] provide practical guidelines and examples for MIMO channel matrix realizations comprising RFC scenarios; [22] treats the formulation and development of the algorithm presented in this chapter; [23-27] detail a splendid survey on architectural aspects for implementing several arithmetic operations.

\section{MIMO systems}

In the context of wireless communication systems, MIMO (Multiple-Input Multiple-Output) is an extension of the classical SISO (Single-Input Single-Output) communication paradigm, 
where instead of having a communication link composed of a single transmitter-end and a receiver-end element (or antenna), wireless MIMO communication systems (or just MIMO systems) consist of an array of multiple elements at both the transmission and reception parts [11-16,19-21]. Generally speaking, the MIMO communication link contains $n_{T}$ transmitter-end and $n_{R}$ receiver-end antennas sending-and-receiving information through a wireless channel. Extensive studies on MIMO systems and commercial devices already employing them reveal that these communication systems offer promising results in terms of: a) spectral efficiency and channel capacity enhancements (many user-end applications supporting high-data rates at limited available bandwidth); b) improvements on Bit-ErrorRate (BER) performance; and c) practical feasability already seen in several wireless communication standards. The conceptualization of this paradigm is illustrated in figure 1, where Tx is the transmitter-end, Rx the receiver-end, and Chx the channel.

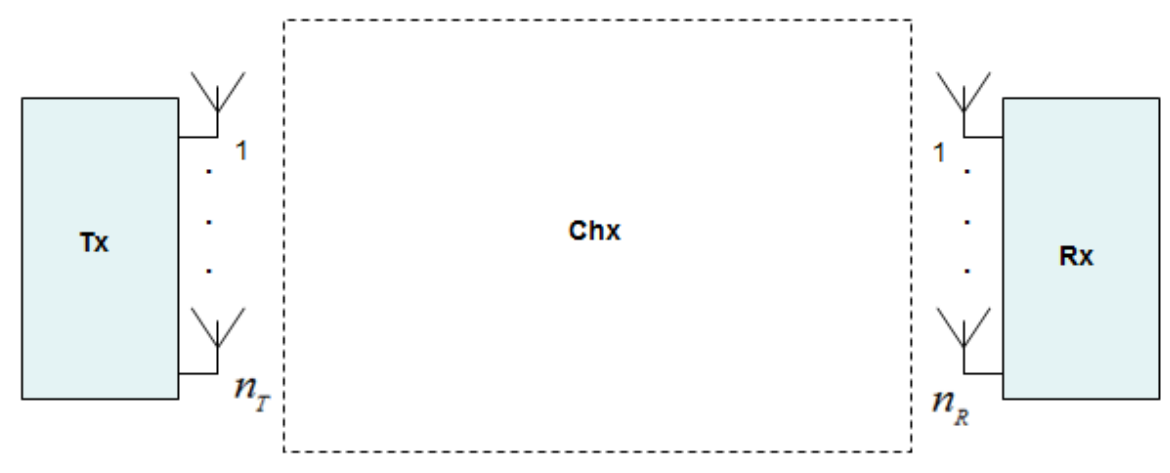

Figure 1. The MIMO system: conceptualization for the MIMO communication paradigm.

Notice that information sent from the trasnmission part (Tx label on figure 1) will suffer from several degradative and distorional effects inherent in the channel (Chx label on figure 1 ), forcing the reception part (Rx label on figure 1) to decode information properly. Information at Rx will suffer from degradations caused by time, frequency, and spatial characteristics of the MIMO communication link [11-12,14]. These issues are directly related to: i) the presence of physical obstacles obstructing the Line-of-Sight (LOS) between Tx and Rx (existance of non-LOS); ii) time delays between received and transmitted information signals due to Tx and Rx dynamical properties (time-selectivity of Chx); iii) frequency distortion and interference among signal carriers through $\mathrm{Chx}$ (frequency-selectivity of Chx); iv) correlation of information between receiver-end elements. Fading (or fading mutlipath) and noise are the most common destructive phenomena that significantly affect information at Rx [11-16]. Fading is a combination of time-frequency replicas of the trasnmitted information as a con- 
sequence of the MIMO system phenomena i)-iv) exposed before, whereas noise affects information at every receiver-end element under an additve or multiplicative way. As a consequence, degradation of signal information rests mainly upon magnitude attenuation and time-frequency shiftings. The simplest treatable MIMO communication link has a slowflat quasi-static fading channel (proper of a non-LOS indoor environment). For this type of scenario, a well-known dynamical-stochastic model considers a Rayleigh fading channel (RFC) [13,15-16,19-21], which gives a quantitative clue of how information has been degradated by means of Chx. Moreover, this type of channels allows to: a) distiguish among each information block tranmitted from the $n_{T}$ elements at every Chx realization (i.e. the time during which the channel's properties remain unvariant); and b) implement easily symbol decoding tasks related to channel equalization (CE) techniques. Likewise, noise is commonly assumed to have additive effects over Rx. Once again, all of these assumptions provide a treatable information-decoding problem (refered as MIMO demodulation [12]), and the mathematical model that suits the aforementioned MIMO communication link characteristics will be represented by

$$
y=H x+\eta
$$

where: $x \in \mathbb{Z}_{[j]}^{n_{T} \times 1} \subset \mathbb{C}^{n_{T} \times 1}$ is a complex-valued $n_{T}$ - dimensional transmitted vector with entries drawn from a Gaussian-integer finite-lattice constellation (digital modulators, such as: $q$-QAM, QPSK); $y \in \mathbb{C}^{n_{R} \times 1}$ is a complex-valued $n_{R}$ - dimensional received vector; $\eta \in \mathbb{C}^{n_{R} \times 1}$ is a $n_{R}$-dimensional independent-identically-distributed (idd) complex-circularly-symmetric (ccs) Additive White Gaussian Noise (AWGN) vector; and $H \in \mathbb{C}^{n_{R} \times n_{T}}$ is the $\left(n_{R} \times n_{T}\right)$ - dimensional MIMO channel matrix whose entries model: a) the RFC-based environment behavior according to a Gaussian probabilistic density function with zero-mean and 0.5variance statistics; and b) the time-invariant transfer function (which measures the degradation of the signal information) between the $i$-th receiver-end and the $j$-th trasnmitterend antennas [11-16,19-21]. Figure 2 gives a representation of (1). As shown therein, the MIMO communication link model stated in (1) can be also expressed as

$$
\left[\begin{array}{l}
y_{1} \\
\vdots \\
y_{n_{R}}
\end{array}\right]=\left[\begin{array}{ccc}
h_{11} & \cdots & h_{1 n_{T}} \\
\vdots & & \vdots \\
h_{n_{R} 1} & \cdots & h_{n_{R} n_{T}}
\end{array}\right]\left[\begin{array}{l}
x_{1} \\
\vdots \\
x_{n_{T}}
\end{array}\right]+\left[\begin{array}{l}
\eta_{1} \\
\vdots \\
\eta_{n_{R}}
\end{array}\right]
$$

Notice from (1-2) that an important requisite for CE purposes within RFC scenarios is that $H$ is provided somehow to the Rx. This MIMO system requirement is classically known as Channel State Information (CSI) [11-16]. In the sequel of this work, symbol-decoding efforts will consider the problem of finding $x$ from $y$ regarding CSI at the Rx part within a slow-flat quasi-static RFC-based environment as modeled in (1-2). In simpler words, Rx must find $x$ from degradated information $y$ through calculating an inversion over $H$. Moreover, $n_{R} \geq n_{T}$ is 
commonly assumed for MIMO demodulation tasks [13-14] because it guarantees linear independency between row-entries of matrix $H$ in (2), yielding a nonhomogeneous overdetermined system of linear equations.

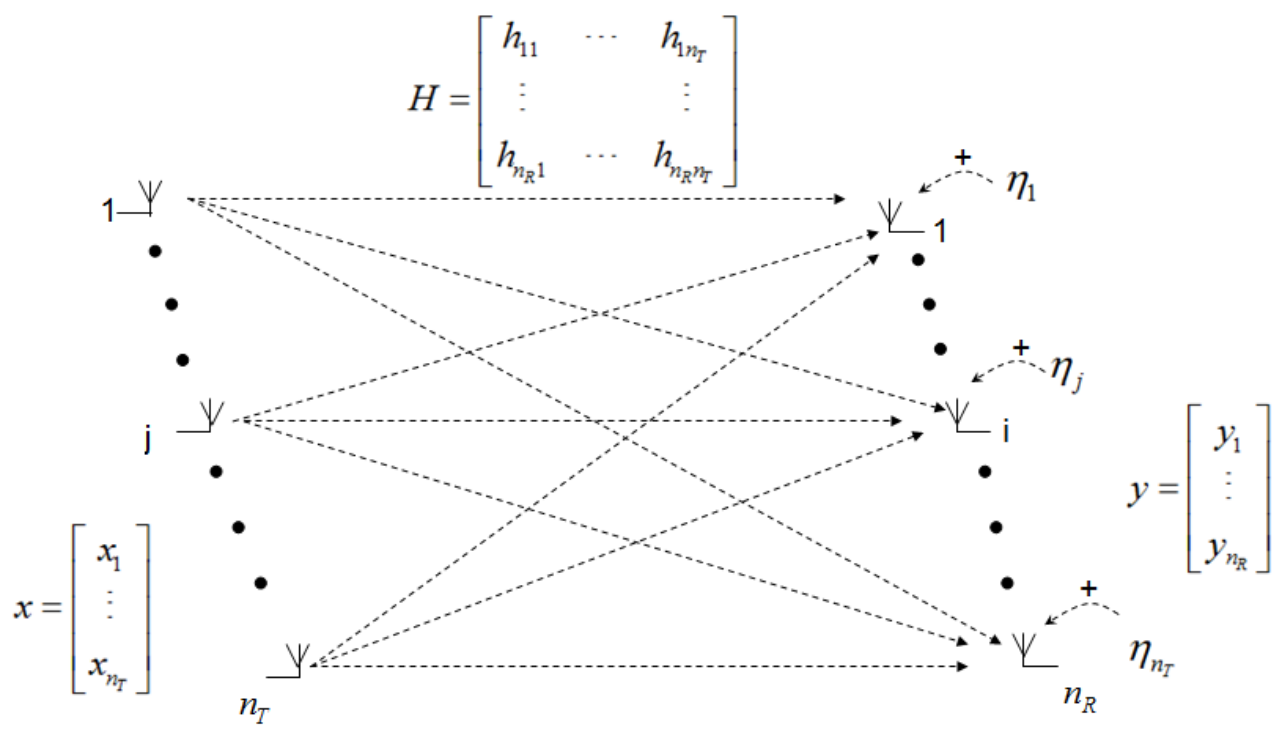

Figure 2. Representation for the MIMO communication link model according to $y=H x+\eta$. Here, each dotted arrow represents an entry $h_{\mathrm{ij}}$ in $H$ which determines channel degradation between the $\mathrm{j}$-th transmitter and the i-th receiver elements. AWGN appears additively in each receiver-end antenna.

\section{Problem definition}

Recall for the moment the mathematical model provided in (1). Consider $\Phi^{\mathrm{r}}$ and $\Phi^{\mathrm{i}}$ to be the real and imaginary parts of a complex-valued matrix (vector) $\Phi$, that is, $\Phi=\Phi^{\mathrm{r}}+j \Phi^{\mathrm{i}}$. Then, Equation (1) can be expanded as follows:

$$
y^{\mathrm{r}}+j y^{\mathrm{i}}=\left(H^{\mathrm{r}} x^{\mathrm{r}}-H^{\mathrm{i}} x^{\mathrm{i}}+\eta^{\mathrm{r}}\right)+j\left(H^{\mathrm{i}} x^{\mathrm{r}}+H^{\mathrm{r}} x^{\mathrm{i}}+\eta^{\mathrm{i}}\right)
$$

It can be noticed from Equation (3) that: $x^{\mathrm{r}}, x^{\mathrm{i}} \in \mathbb{Z}^{n_{\mathrm{T}} \times 1} ; y^{\mathrm{r}}, y^{\mathrm{i}} \in \mathbb{R}^{n_{R} \times 1} ; \eta^{\mathrm{r}}, \eta^{\mathrm{i}} \in \mathbb{R}^{n_{\mathrm{R}} \times 1}$; and $H^{\mathrm{r}}, H^{\mathrm{i}} \in \mathbb{R}^{n_{R} \times n_{T}}$. An alternative representation for the MIMO communication link model in (2) can be expressed as 


$$
\left[\begin{array}{l}
y^{\mathrm{r}} \\
y^{\mathrm{i}}
\end{array}\right]=\left[\begin{array}{cc}
H^{\mathrm{r}} & -H^{\mathrm{i}} \\
H^{\mathrm{i}} & H^{\mathrm{r}}
\end{array}\right]\left[\begin{array}{l}
x^{\mathrm{r}} \\
x^{\mathrm{i}}
\end{array}\right]+\left[\begin{array}{c}
\eta^{\mathrm{r}} \\
\eta^{\mathrm{i}}
\end{array}\right]
$$

where $\left[\begin{array}{l}y^{\mathrm{r}} \\ y^{\mathrm{i}}\end{array}\right] \doteq \mathrm{Y} \in \mathbb{R}^{2 n_{R} \times 1}, \quad\left[\begin{array}{cc}H^{\mathrm{r}} & -H^{\mathrm{i}} \\ H^{\mathrm{i}} & H^{\mathrm{r}}\end{array}\right] \doteq \mathrm{h} \in \mathbb{R}^{2 n_{R} \times 2 n_{T}}, \quad\left[\begin{array}{l}x^{\mathrm{r}} \\ x^{\mathrm{i}}\end{array}\right] \doteq \mathrm{X} \in \mathbb{Z}^{2 n_{T} \times 1}, \quad$ and $\left[\begin{array}{l}\eta^{\mathrm{r}} \\ \eta^{\mathrm{i}}\end{array}\right] \doteq \mathrm{N} \in \mathbb{R}^{2 n_{R} \times 1}$. CSI is still needed for MIMO demodulation purposes involving (4). Moreover, if $N_{r}=2 n_{R}$ and $N_{t}=2 n_{T}$, then $N_{r} \geq N_{t}$. Obviously, while seeking for a solution of signal vector $\mathrm{X}$ from (4), the reception part $\mathrm{Rx}$ will provide also the solution for signal vector $x$, and thus MIMO demodulation tasks will be fulfilled. This problem can be defined formally into the following manner:

Definition 1. Given parameters $N_{r}=2 n_{R} \in \mathbb{Z}^{+}$and $N_{t}=2 n_{T} \in \mathbb{Z}^{+}$, and a block-matrix $\mathrm{h} \in \mathbb{R}^{N_{r} \times N_{t}}$, there exists an operator $\Gamma:\left(\mathbb{R}^{N_{r} \times 1} \times \mathbb{R}^{\left.N_{r} \times N_{t}\right)} \mapsto \mathbb{R}^{N_{t} \times 1}\right.$ which solves the matrixblock equation $\mathrm{Y}=\mathrm{h} \mathrm{X}+\mathrm{N}$ so that $\Gamma[\mathrm{Y}, \mathrm{h}]=\mathrm{X}$.

From Definition 1, the following affirmations hold: i) CSI over his a necessary condition as an input argument for the operator $\Gamma$; and ii) $\Gamma$ can be naïvely defined as a Generalized-Inverse of the block-matrixh. In simpler terms, $\mathrm{X}=\mathrm{h}^{\dagger} \mathrm{Y}^{1}$ is associated with $\Gamma[\mathrm{Y}, \mathrm{h}]$ and $\mathrm{h}^{\dagger} \in \mathbb{R}^{N_{t} \times N_{r}}$ stands for the Generalized-Inverse of the block-matrixh, where $\mathrm{h}^{\dagger}=\left(\mathrm{h}^{\mathrm{T}} \mathrm{h}\right)^{-1} \mathrm{~h}^{\mathrm{T}}$ [17-18]. Clearly, []$^{-1}$ and []$^{\mathrm{T}}$ represent the inverse and transpose matrix operations over realvalued matrices. As a concluding remark, computing the Generalized-Inverse $\mathrm{h}^{\dagger}$ can be separated into two operations: 1) a block-matrix inversion $\left(\mathrm{h}^{\mathrm{T}} \mathrm{h}\right)^{-1_{2}}$; 2) a typical matrix multiplication $\left(h^{T} h\right)^{-1} \cdot h^{T}$. For these tasks, Partition-Matrix Theory will be employed in order to find a novel algorithm for computing a Generalized-Inverse related to (4).

\section{Mathematical background}

\subsection{Partition-matrix theory}

Partition-Matrix Theory embraces structures related to block matrices (or partition matrices: an array of matrices) [17-18]. Furthermore, a block-matrix $L$ with $(n+q) \times(m+p)$ dimension can be constructed (or partitioned) consistently according to matrix sub-blocks $A, B, C$, and $D$ of $n \times m, n \times p, q \times m$, and $q \times p$ dimensions, respectively, yielding

1 In the context of MIMO systems, this matrix operation is commonly found in Babai estimators for symbol-decoding purposes at the Rx part [12,13]. For the reader's interest, refer to [11-16] for other MIMO demodulation techniques.

2 Notice that and. 


$$
L=\left[\begin{array}{ll}
A & B \\
C & D
\end{array}\right]
$$

An interesting operation to be performed for these structures given in (5) is the inversion, i.e. a blockwise inversion $L^{-1}$. For instance, let $L \in \mathbb{R}^{(n+m) \times(n+m)}$ be a full-rank real-valued block matrix (the subsequent treatment is also valid for complex-valued entities, i.e. $\left.L \in \mathbb{C}^{(n+m) \times(n+m)}\right)$. An alternative partition can be performed with $A \in \mathbb{R}^{n \times n}, B \in \mathbb{R}^{n \times m}$, $C \in \mathbb{R}^{m \times n}$, and $D \in \mathbb{R}^{m \times m}$. Assume also $A$ and $D$ to be full-rank matrices. Then,

$$
L^{-1}=\left[\begin{array}{cc}
\left(A-B D^{-1} C\right)^{-1} & -\left(A-B D^{-1} C\right)^{-1} B D^{-1} \\
-\left(D-C A^{-1} B\right)^{-1} C A^{-1} & \left(D-C A^{-1} B\right)^{-1}
\end{array}\right]
$$

This strategy (to be proved in the next part) requires additonally and mandatorily full-rank over matrices $A-B D^{-1} C$ and $D-C A^{-1} B$. The simple case is defined for $L=\left[\begin{array}{ll}a & b \\ c & d\end{array}\right]$ (indistinctly for $\mathbb{R}^{2 \times 2}$ or $\mathbb{C}^{2 \times 2}$ ). Once again, assumingdet $(L) \neq 0, a \neq 0$, and $d \neq 0$ (related to full-rank restictions within block-matrix $L$ ):

$L^{-1}=\left[\begin{array}{cc}\left(a-b d^{-1} c\right)^{-1} & -\left(a-b d^{-1} c\right)^{-1} b d^{-1} \\ -\left(d-c a^{-1} b\right)^{-1} c a^{-1} & \left(d-c a^{-1} b\right)^{-1}\end{array}\right]=\frac{1}{a d-b c}\left[\begin{array}{cc}d & -b \\ -c & a\end{array}\right]$,

where evidently $(a d-b c) \neq 0, \mathbb{R} \mathbb{C}^{(n+m) \times(n+m)}\left(a-b d^{-1} c\right) \neq 0$, and $\left(d-c a^{-1} b\right) \neq 0$.

\subsection{Matrix Inversion Lemma}

The Matrix Inversion Lemma is an indirect consequence of inverting non-singular block matrices [17-18], either real-valued or complex-valued, e.g., under certain restrictions ${ }^{3}$. Lemma 1 states this result.

Lemma 1. Let $\Psi \in \mathbb{R} \mathbb{C}^{r \times r}, \Sigma \in \mathbb{R} \mathbb{C}^{r \times s}, \in \mathbb{R} \mathbb{C}^{s \times s}$, and $\Xi \in \mathbb{R} \mathbb{C}^{s \times r}$ be real-valued or complex-valued matrices. Assume these matrices to be non-singular: $\Psi,(\Psi+\Sigma \Xi)$, and $\left({ }^{-1}+\Xi \Psi^{-1} \Sigma\right)$. Then,

$$
(\Psi+\Sigma \Xi)^{-1}=\Psi^{-1}-\Psi^{-1} \Sigma\left({ }^{-1}+\Xi \Psi^{-1} \Sigma\right)^{-1} \Xi \Psi^{-1}
$$

Proof. The validation of (7) must satisfy

i.

$$
(\Psi+\Sigma \Xi) \cdot\left(\Psi^{-1}-\Psi^{-1} \Sigma\left({ }^{-1}+\Xi \Psi^{-1} \Sigma\right)^{-1} \Xi \Psi^{-1}\right)=I_{r}
$$

3 Refer to $[3,7-10,17,18]$ to review lemmata exposed for these issues and related results. 
$\left(\Psi^{-1}-\Psi^{-1} \Sigma\left({ }^{-1}+\Xi \Psi^{-1} \Sigma\right)^{-1} \Xi \Psi^{-1}\right) \cdot(\Psi+\Sigma \Xi)=I_{r}$, where $I_{r}$ represents the $r \times r$ identity matrix. Notice the existance of matrices $\Psi^{-1},{ }^{-1},(\Psi+\Sigma \Xi)^{-1}$ and $\left({ }^{-1}+\Xi \Psi^{-1} \Sigma\right)^{-1}$. Manipulating i) shows:

$$
\begin{aligned}
& (\Psi+\Sigma \Xi) \cdot\left(\Psi^{-1}-\Psi^{-1} \Sigma\left({ }^{-1}+\Xi \Psi^{-1} \Sigma\right)^{-1} \Xi \Psi^{-1}\right) \\
& =I_{r}-\Sigma\left({ }^{-1}+\Xi \Psi^{-1} \Sigma\right)^{-1} \Xi \Psi^{-1}+\Sigma \Xi \Psi^{-1}-\Sigma \Xi \Psi^{-1} \Sigma\left({ }^{-1}+\Xi \Psi^{-1} \Sigma\right)^{-1} \Xi \Psi^{-1} \\
& =I_{r}+\Sigma \Xi \Psi^{-1}-\Sigma\left(^{-1}+\Xi \Psi^{-1} \Sigma\right)\left({ }^{-1}+\Xi \Psi^{-1} \Sigma\right)^{-1} \Xi \Psi^{-1} \\
& =I_{r}+\Sigma \Xi \Psi^{-1}-\Sigma \Xi \Psi^{-1}=I_{r} .
\end{aligned}
$$

Likewise for ii):

$$
\begin{aligned}
& \left(\Psi^{-1}-\Psi^{-1} \Sigma\left({ }^{-1}+\Xi \Psi^{-1} \Sigma\right)^{-1} \Xi \Psi^{-1}\right) \cdot(\Psi+\Sigma \Xi) \\
& =I_{r}+\Psi^{-1} \Sigma \Xi-\Psi^{-1} \Sigma\left({ }^{-1}+\Xi \Psi^{-1} \Sigma\right)^{-1} \Xi-\Psi^{-1} \Sigma\left(^{-1}+\Xi \Psi^{-1} \Sigma\right)^{-1} \Xi \Psi^{-1} \Sigma \Xi \\
& =I_{r}+\Psi^{-1} \Sigma \Xi-\Psi^{-1} \Sigma\left({ }^{-1}+\Xi \Psi^{-1} \Sigma\right)^{-1}\left({ }^{-1}+\Xi \Psi^{-1} \Sigma\right) \Xi \\
& =I_{r}+\Psi^{-1} \Sigma \Xi-\Psi^{-1} \Sigma \Xi=I_{r} .
\end{aligned}
$$

Now it is pertinent to demonstrate (6) with the aid of Lemma 1. It must be verified that both $L L^{-1}$ and $L^{-1} L$ must be equal to the $(n+m) \times(n+m)$ identity block matrix $I_{(n+m)}=\left[\begin{array}{cc}I_{n} & 0_{n \times m} \\ 0_{m \times n} & I_{m}\end{array}\right]$, with consistent-dimensional identity and zero sub-blocks: $I_{n}, I_{m} ; 0_{n \times m}$ $0_{m \times n}$, respectively. We start by calulating

$$
L L^{-1}=\left[\begin{array}{ll}
A & B \\
C & D
\end{array}\right]\left[\begin{array}{cc}
\left(A-B D^{-1} C\right)^{-1} & -\left(A-B D^{-1} C\right)^{-1} B D^{-1} \\
-\left(D-C A^{-1} B\right)^{-1} C A^{-1} & \left(D-C A^{-1} B\right)^{-1}
\end{array}\right]
$$

and

$$
L^{-1} L=\left[\begin{array}{cc}
\left(A-B D^{-1} C\right)^{-1} & -\left(A-B D^{-1} C\right)^{-1} B D^{-1} \\
-\left(D-C A^{-1} B\right)^{-1} C A^{-1} & \left(D-C A^{-1} B\right)^{-1}
\end{array}\right]\left[\begin{array}{cc}
A & B \\
C & D
\end{array}\right]
$$

by applying (7) in Lemma 1 to both matrices $\left(A-B D^{-1} C\right)^{-1} \in \mathbb{R} \mathbb{C}^{n \times n}$ and $\left(D-C A^{-1} B\right)^{-1} \in \mathbb{R} \mathbb{C}^{m \times m}$, which are present in (8) and (9), and recalling full-rank conditions not only over those matrices but also for $A$ and $D$, yields the relations

$$
\left(A-B D^{-1} C\right)^{-1}=A^{-1}+A^{-1} B\left(D-C A^{-1} B\right)^{-1} C A^{-1}
$$




$$
\left(D-C A^{-1} B\right)^{-1}=D^{-1}+D^{-1} C\left(A-B D^{-1} C\right)^{-1} B D^{-1}
$$

Using (10-11) in (8-9), the following results arise:

a. for operations involved in sub-blocks of $L L^{-1}$ :

$$
\begin{aligned}
& A\left(A-B D^{-1} C\right)^{-1}-B\left(D-C A^{-1} B\right)^{-1} C A^{-1} \\
& =A\left[A^{-1}+A^{-1} B\left(D-C A^{-1} B\right)^{-1} C A^{-1}\right]-B\left(D-C A^{-1} B\right)^{-1} C A^{-1} \\
& =I_{n}+B\left(D-C A^{-1} B\right)^{-1} C A^{-1}-B\left(D-C A^{-1} B\right)^{-1} C A^{-1}=I_{n} \\
& -A\left(A-B D^{-1} C\right)^{-1} B D^{-1}+B\left(D-C A^{-1} B\right)^{-1} \\
& =-A\left[A^{-1}+A^{-1} B\left(D-C A^{-1} B\right)^{-1} C A^{-1}\right] B D^{-1}+B\left(D-C A^{-1} B\right)^{-1} \\
& =-B D^{-1}-B\left(D-C A^{-1} B\right)^{-1} C A^{-1} B D^{-1}+B\left(D-C A^{-1} B\right)^{-1} \\
& =-B D^{-1}-B\left(D-C A^{-1} B\right)^{-1}\left(-C A^{-1} B+D\right) D^{-1}=0_{n \times m} \\
& C\left(A-B D^{-1} C\right)^{-1}-D\left(D-C A^{-1} B\right)^{-1} C A^{-1} \\
& =C\left(A-B D^{-1} C\right)^{-1}-D\left[D^{-1}+D^{-1} C\left(A-B D^{-1} C\right)^{-1} B D^{-1}\right] C A^{-1} \\
& =C\left(A-B D^{-1} C\right)^{-1}-C A^{-1}-C\left(A-B D^{-1} C\right)^{-1} B D^{-1} C A^{-1} \\
& =C\left(A-B D^{-1} C\right)^{-1}\left[A-B D^{-1} C\right] A^{-1}-C A^{-1}=0_{m \times n} ; \\
& -C\left(A-B D^{-1} C\right)^{-1} B D^{-1}+D\left(D-C A^{-1} B\right)^{-1} \\
& =-C\left(A-B D^{-1} C\right)^{-1} B D^{-1}+D\left[D^{-1}+D^{-1} C\left(A-B D^{-1} C\right)^{-1} B D^{-1}\right] \\
& =-C\left(A-B D^{-1} C\right)^{-1} B D^{-1}+I_{m}+C\left(A-B D^{-1} C\right)^{-1} B D^{-1}=I_{m^{\prime}}
\end{aligned}
$$

thus,

$$
L L^{-1}=I_{(n+m)}
$$

2. for operations involved in sub-blocks of $L^{-1} L$ :

$$
\begin{aligned}
& \left(A-B D^{-1} C\right)^{-1} A-\left(A-B D^{-1} C\right)^{-1} B D^{-1} C \\
& =\left(A-B D^{-1} C\right)^{-1}\left[A-B D^{-1} C\right]=I_{n} ; \\
& \left(A-B D^{-1} C\right)^{-1} B-\left(A-B D^{-1} C\right)^{-1} B D^{-1} D=0_{n \times m} ;
\end{aligned}
$$




$$
\begin{aligned}
& -\left(D-C A^{-1} B\right)^{-1} C A^{-1} A+\left(D-C A^{-1} B\right)^{-1} C=0_{m \times n} ; \\
& -\left(D-C A^{-1} B\right)^{-1} C A^{-1} B+\left(D-C A^{-1} B\right)^{-1} D \\
& =-\left(D-C A^{-1} B\right)^{-1}\left[-C A^{-1} B+D\right]=I_{m} ;
\end{aligned}
$$

thus, $L^{-1} L=I_{(n+m)}$.

\subsection{Generalized-Inverse}

The concept of Generalized-Inverse is an extension of a matrix inversion operations applied to non-singular rectangular matrices [17-18]. For notation purposes and without loss of generalization, $\rho(G)$ and $G^{T}$ denote the rank of a rectangular matrix $G \in \mathrm{M}^{m \times n}$, and $G^{T}=G^{\mathrm{H}}$ is the transpose-conjugate of $G$ (whenM= $C \rightarrow G \in \mathbb{C}^{m \times n}$ ) or $G^{T}=G^{T}$ is the transpose of $G$ (whenM= $\mathbb{R} \rightarrow G \in \mathbb{R}^{m \times n}$ ), respectively.

Definition 2. Let $G \in \mathrm{M}^{m \times n}$ and $0 \leq \rho(G) \leq \min (m, n)$. Then, there exists a matrix $G^{\dagger} \in \mathrm{M}^{n \times m}$ (identified as the Generalized-Inverse), such that it satisfies several conditions for the following cases:

case i: if $m>n$ and $0 \leq \rho(G) \leq \min (m, n) \Rightarrow \rho(G)=n$, then there exists a unique matrix $G^{\dagger} \doteq G^{+} \in \mathrm{M}^{n \times m}$ (identified as Left-Pseudoinverse: LPI) such that $G^{+} G=I_{n^{\prime}}$ satisfying: a) $G G^{+} G=G$, and $\left.b\right) G^{+} G G^{+}=G^{+}$. Therefore, the LPI matrix is proposed as $G^{+}=\left(G^{T} G\right)^{-1} G^{T}$.

case ii: if $m=n$ anddet $(G) \neq 0 \Leftrightarrow \rho(G)=n$, then there exists a unique matrix $G^{\dagger} \doteq G^{-1} \in \mathrm{M}^{n \times n}$ (identified as Inverse) such that $G^{-1} G=G G^{-1}=I_{n}$.

case iii: if $m<n$ and $0 \leq \rho(G) \leq \min (m, n) \Rightarrow \rho(G)=m$, then there exists a unique matrix $G^{+} \doteq G^{-} \in \mathrm{M}^{n \times m} \overline{\text { (identified as Right-Pseudoinverse: RPI) such that }} G G^{-}=I_{m^{\prime}} \overline{\text { satisfying: a) }}$ $G G^{-} G=G \overline{\text { and b) }} G^{-} G G^{-}=G^{-}$. Therefore, the RPI matrix is proposed as $G^{-}=G^{T}\left(G G^{T}\right)^{-1}$.

Given the mathematical structure for $G^{+}$provided in Definition 2, it can be easily validated that: 1) For a LPI matrix stipulated in case i, $G G^{\dagger} G=G$ and $G^{\dagger} G G^{\dagger}=G^{\dagger}$ with $\left.G^{\dagger}=\left(G^{T} G\right)^{-1} G^{T} ; 2\right)$ For a RPI matrix stipulated in case iii, $G G^{\dagger} G=G$ and $G^{\dagger} G G^{\dagger}=G^{\dagger}$ with $G^{+}=G^{T}\left(G G^{T}\right)^{-1}$; iii) For the Inverse in case ii, $G^{+}=\left(G^{T} G\right)^{-1} G^{T}=G^{T}\left(G G^{T}\right)^{-1}=G^{-}$. For a uniqueness test for all cases, assume the existance of matrices $G_{1}^{+} \in \mathrm{M}^{n \times m}$ and $G_{2}^{+} \in \mathrm{M}^{n \times m}$ such that $G_{1}^{\dagger} G=I_{n}$ and $G_{2}^{\dagger} G=I_{n}$ (for case i), and $G G_{1}^{\dagger}=I_{m}$ and $G G_{2}^{\dagger}=I_{m}$ (for case iii). Notice immediately, $\left(G_{1}^{\dagger}-G_{2}^{\dagger}\right) G=0_{n}\left(\right.$ for case i) and $G\left(G_{1}^{\dagger}-G_{2}^{\dagger}\right)=0_{m}$ (for case iii), which obligates $G_{1}^{+}=G_{2}^{+}$for both cases, because of full-rank properties over $G$. Clearly, case ii is a particular consequence of cases $i$ and iii. 


\section{The MIMO channel matrix}

The MIMO channel matrix is the mathematical representation for modeling the degradation phenomena presented in the RFC scenario presented in (2). The elements $h_{\mathrm{ij}}$ in $H \in \mathbb{C}^{n_{\mathrm{R}} \times n_{T}}$ represent a time-invariant transfer function (possesing spectral information about magnitude and phase profiles) between a j-th transmitter and an i-th receiver antenna. Once again, dynamical properties of physical phenomena ${ }^{4}$ such as path-loss, shadowing, multipath, Doppler spreading, coherence time, absorption, reflection, scattering, diffraction, basestation-user motion, antenna's physical properties-dimensions, information correlation, associated with a slow-flat quasi-static RFC scenario (proper of a non-LOS indoor wireless environments) are highlighted into a statistical model represented by matrix $H$. For $H^{\dagger}$ purposes, CSI is a necessary feature required at the reception part in (2), as well as the $n_{R} \geq n_{T}$ condition. Table 1 provides several $n_{R}>n_{T}$ MIMO channel matrix realizations for RFC-based environments [19-21]. On table 1: a) $\mathrm{MIMO}\left(n_{R}, n_{T}\right)$ : refers to the MIMO communication link configuration, i.e. amount of receiver-end and transmitter-end elements; $\mathrm{b}) H_{\mathrm{m}}$ : refers to a MIMO channel matrix realization; c) $H_{\mathrm{m}}^{+}$: refers to the corresponding LPI, computed as $\left.H_{\mathrm{m}}^{+}=\left(H_{\mathrm{m}}^{\mathrm{H}} H_{\mathrm{m}}\right)^{-1} H_{\mathrm{m}}^{\mathrm{H}} ; \mathrm{d}\right) \mathrm{h}$ : blockwise matrix version for $\left.H_{\mathrm{m}} ; \mathrm{e}\right) \mathrm{h}^{+}$: refers to the corresponding LPI, computed $a h^{+}=\left(h^{T} h\right)^{-1} h^{T}$. As an additional point of analysis, full-rank properties over $H$ and $\mathrm{h}$ (and thus the existance of matrices $H^{+}, H^{-1}, \mathrm{~h}^{+}$, andh ${ }^{-1}$ ) are validated and corroborated through a MATLAB simulation-driven model regarding frequency-selective and timeinvariant properties for several RFC-based scenarios at different MIMO configurations. Experimental data were generated upon $10^{6} \mathrm{MIMO}$ channel matrix realizations. As illustrated in figure 3, a common pattern is found regarding the statistical evolution for full-rank properties of $H$ and $\mathrm{h}$ with $n_{R} \geq n_{T}$ at several typical MIMO configurations, for instance, $\operatorname{MIMO}(2,2), \operatorname{MIMO}(4,2)$, $\operatorname{andMIMO}(4,4)$. It is plotted therein $\operatorname{REAL}(\mathrm{H}, \mathrm{h})$ against $\operatorname{IMAG}(\mathrm{H}, \mathrm{h})$, where each axis label denote respectively the real and imaginary parts of: a) $\operatorname{det}(H)$ and $\operatorname{det}(\mathrm{h}) w \operatorname{when} n_{R}=n_{T}$, and $\left.\mathrm{b}\right) \operatorname{det}\left(H^{\mathrm{H}} H\right)$ and $\operatorname{det}\left(\mathrm{h}^{\mathrm{T}} \mathrm{h}\right)$ when. Blue crosses indicate the behavior of $\rho(H)$ related to $\operatorname{det}(H)$ and $\operatorname{det}\left(H^{\mathrm{H}} H\right)(\operatorname{det}(\mathrm{H})$ legend on top-left margin), while red crosses indicate the behavior of $\rho(\mathrm{h})$ related to $\operatorname{det}(\mathrm{h})$ and $\operatorname{det}\left(\mathrm{h}^{\mathrm{T}} \mathrm{h}\right)(\operatorname{det}(\mathrm{h}) \operatorname{legend}$ on top-left margin). The black-circled zone intersected with black-dotted lines locates the $0+j 0$ value. As depicted on figures (4)-(5), a closer glance at this statistical behavior reveals a prevalence on full-rank properties of $H$ andh, meaning that non of the determinants $\operatorname{det}(H), \operatorname{det}(\mathrm{h}), \operatorname{det}\left(H^{\mathrm{H}} H\right)$ and $\operatorname{det}\left(\mathrm{h}^{\mathrm{T}} \mathrm{h}\right)$ is equal to zero (behavior enclosed by the light-blue region and delimited by blue/red-dotted lines). 


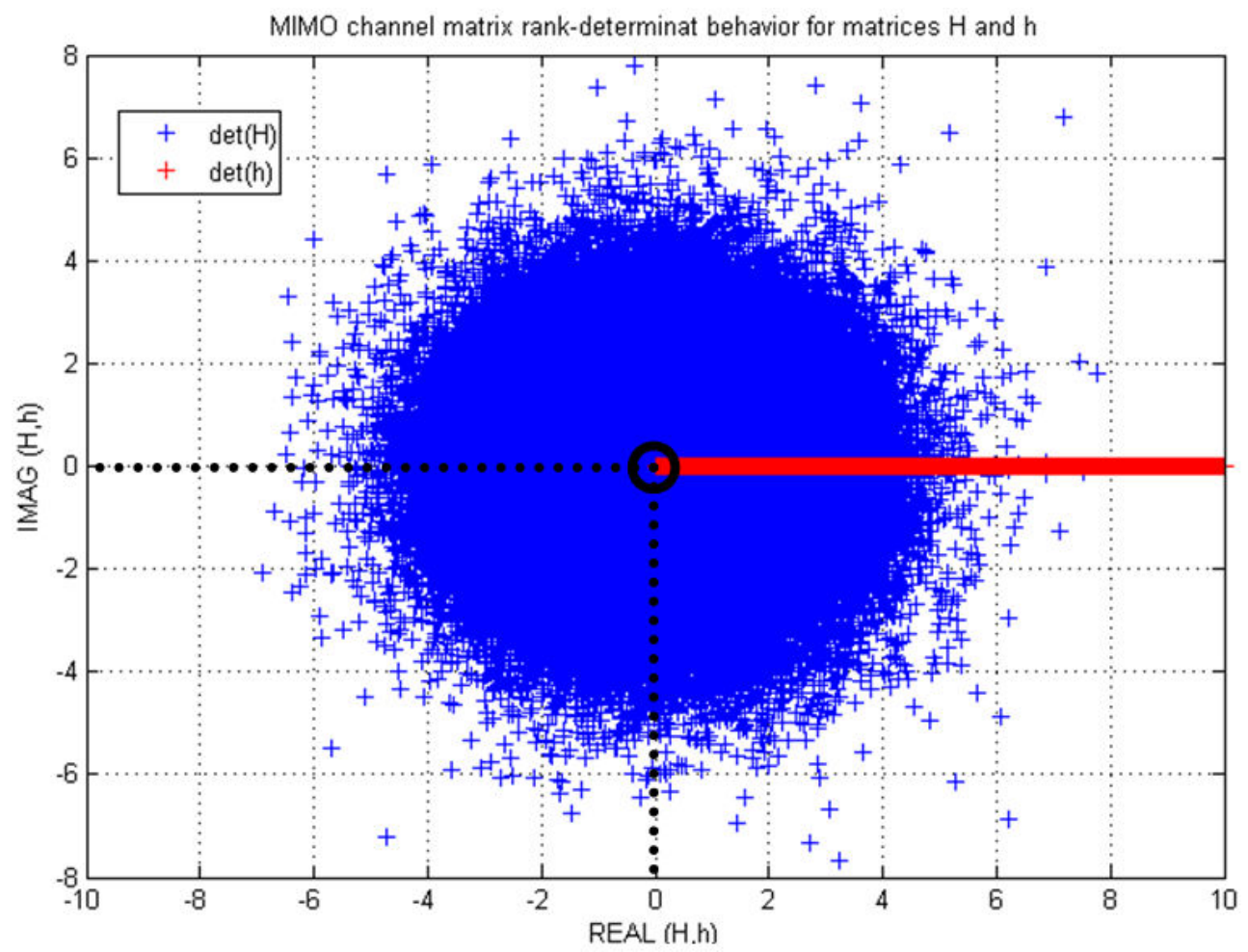

Figure 3. MIMO channel matrix rank-determinant behavior for several realizations for $H$ andh. This statistical evolution is a common pattern found for several MIMO configurations involving slow-flat quasi-static RFC-based environments with $n_{R} \geq n_{T}$. 


\begin{tabular}{|c|c|c|c|c|c|c|c|c|c|}
\hline $\operatorname{MMO}\left(n_{R}, n_{\tau}\right)$ & \multicolumn{4}{|c|}{$H_{\mathrm{m}}$} & \multicolumn{5}{|c|}{$H_{\mathrm{m}}^{+}$} \\
\hline $\operatorname{MIMO}(2,2)$ & & {$\left[\begin{array}{l}1.12 \\
0.23\end{array}\right.$} & $\left.\begin{array}{l}0.15 \\
0.96\end{array}\right]$ & & & {$\left[\begin{array}{c}0.92 \\
-0.22\end{array}\right.$} & $\begin{array}{ll}2 & -0.1 \\
1 & 1.0\end{array}$ & $\left.\begin{array}{l}144 \\
76\end{array}\right]$ & \\
\hline $\operatorname{MIMO}(4,2)$ & {$\left[\begin{array}{c}0.85- \\
-0.37 \\
-0.06 \\
-0.91\end{array}\right.$} & $\begin{array}{l}-j 0.47 \\
-j 0.72 \\
+j 0.45 \\
+j 1.03\end{array}$ & $\begin{array}{r}-0.06 \\
0.94 \\
1.14 \\
1.07\end{array}$ & $\left.\begin{array}{l}6+j 0.34 \\
-j 0.28 \\
+j 1.13 \\
-j 0.02\end{array}\right]$ & {$\left[\begin{array}{r}0.256+ \\
0.051- \\
0.0 \\
0.23\end{array}\right.$} & $\begin{array}{l}+j 0.115 \\
-j 0.082 \\
088-j 0 \\
234-j 0\end{array}$ & 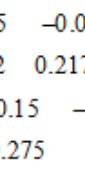 & $\begin{array}{c}06+j 0.2 \\
7+j 0.1 \\
-0.206- \\
0.15-j\end{array}$ & $\left.\begin{array}{l}26 \\
116 \\
-j 0.258 \\
j 0.018\end{array}\right]$ \\
\hline $\operatorname{MMO}\left(n_{R}, n_{f}\right)$ & \multicolumn{4}{|c|}{$\mathrm{h}$} & \multicolumn{5}{|c|}{$\mathrm{h}^{+}$} \\
\hline $\operatorname{MIMO}(2,2)$ & {$\left[\begin{array}{c}1.12 \\
0.23 \\
0 \\
0\end{array}\right.$} & $\begin{array}{c}0.15 \\
0.96 \\
0 \\
0\end{array}$ & $\begin{array}{c}0 \\
0 \\
1.12 \\
0.23\end{array}$ & $\left.\begin{array}{c}0 \\
0 \\
0.15 \\
0.96\end{array}\right]$ & {$\left[\begin{array}{c}0.922 \\
-0.22 \\
0 \\
0\end{array}\right.$} & $\begin{array}{rr}2 & -0.1 \\
1.07 \\
0 \\
0\end{array}$ & $\begin{array}{rr}44 & 0 \\
6 & 0 \\
& 0.9 \\
& -0.2\end{array}$ & $\begin{array}{ll}0 & \\
0 & \\
922 & -0 . \\
221 & 1.0\end{array}$ & $\left.\begin{array}{l}0 \\
0 \\
144 \\
076\end{array}\right]$ \\
\hline $\operatorname{MIMO}(4,2)$ & {$\left[\begin{array}{c}0.85 \\
-0.37 \\
-0.06 \\
-0.91 \\
-0.47 \\
-0.72 \\
0.45 \\
1.03\end{array}\right.$} & $\begin{array}{c}-0.06 \\
0.94 \\
1.14 \\
1.07 \\
0.34 \\
-0.28 \\
1.13 \\
-0.02\end{array}$ & $\begin{array}{c}0.47 \\
0.72 \\
-0.45 \\
-1.03 \\
0.85 \\
-0.37 \\
-0.06 \\
-0.91\end{array}$ & $\left.\begin{array}{c}-0.34 \\
0.28 \\
-1.13 \\
0.02 \\
-0.06 \\
0.94 \\
1.14 \\
1.07\end{array}\right]$ & {$\left[\begin{array}{c}0.256 \\
0.051 \\
0.115 \\
-0.082\end{array}\right.$} & $\begin{array}{c}-0.06 \\
0.217 \\
0.26 \\
0.116 \\
-0.115 \\
0.082 \\
0.256 \\
0.051\end{array}$ & $\begin{array}{l}0.088 \\
0.234 \\
-0.15 \\
-0.275 \\
-0.26 \\
-0.116 \\
-0.06 \\
0.217\end{array}$ & $\begin{array}{l}-0.206 \\
0.15 \\
-0.258 \\
-0.018 \\
0.15 \\
0.275 \\
0.088 \\
0.234\end{array}$ & $\left.\begin{array}{c}0.258 \\
0.018 \\
-0.206 \\
0.15\end{array}\right]$ \\
\hline
\end{tabular}

Table 1. MIMO channel matrix realizations for several MIMO communication link configurations at slow-flat quasistatic RFC scenarios. 


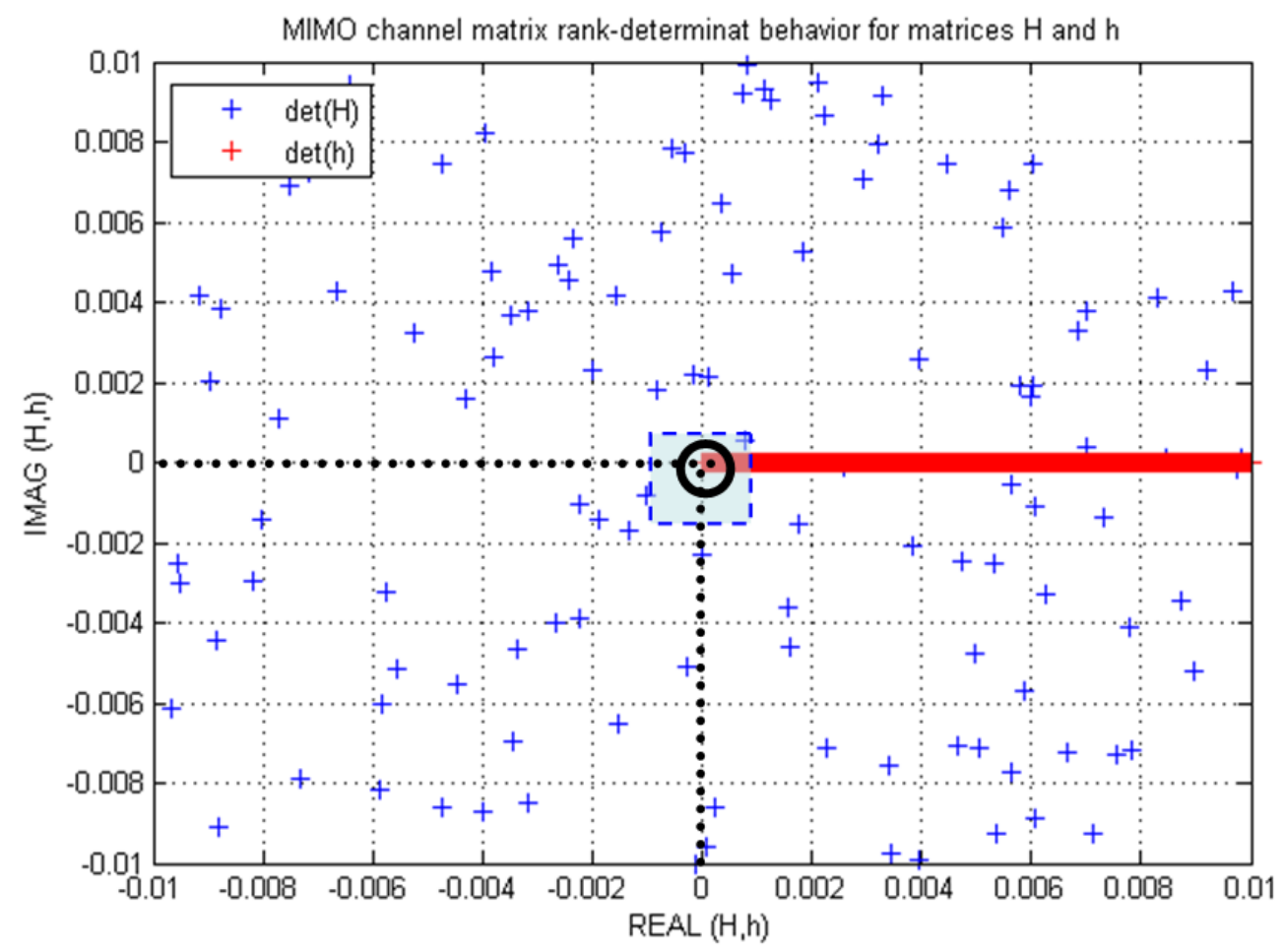

Figure 4. MIMO channel matrix rank-determinant behavior for several realizations for $H$. Full-rank properties for $H$ and $\mathrm{H}^{\mathrm{H}} \mathrm{H}$ preveal for RFC-based environments (light-blue region delimited by blue-dotted lines). 


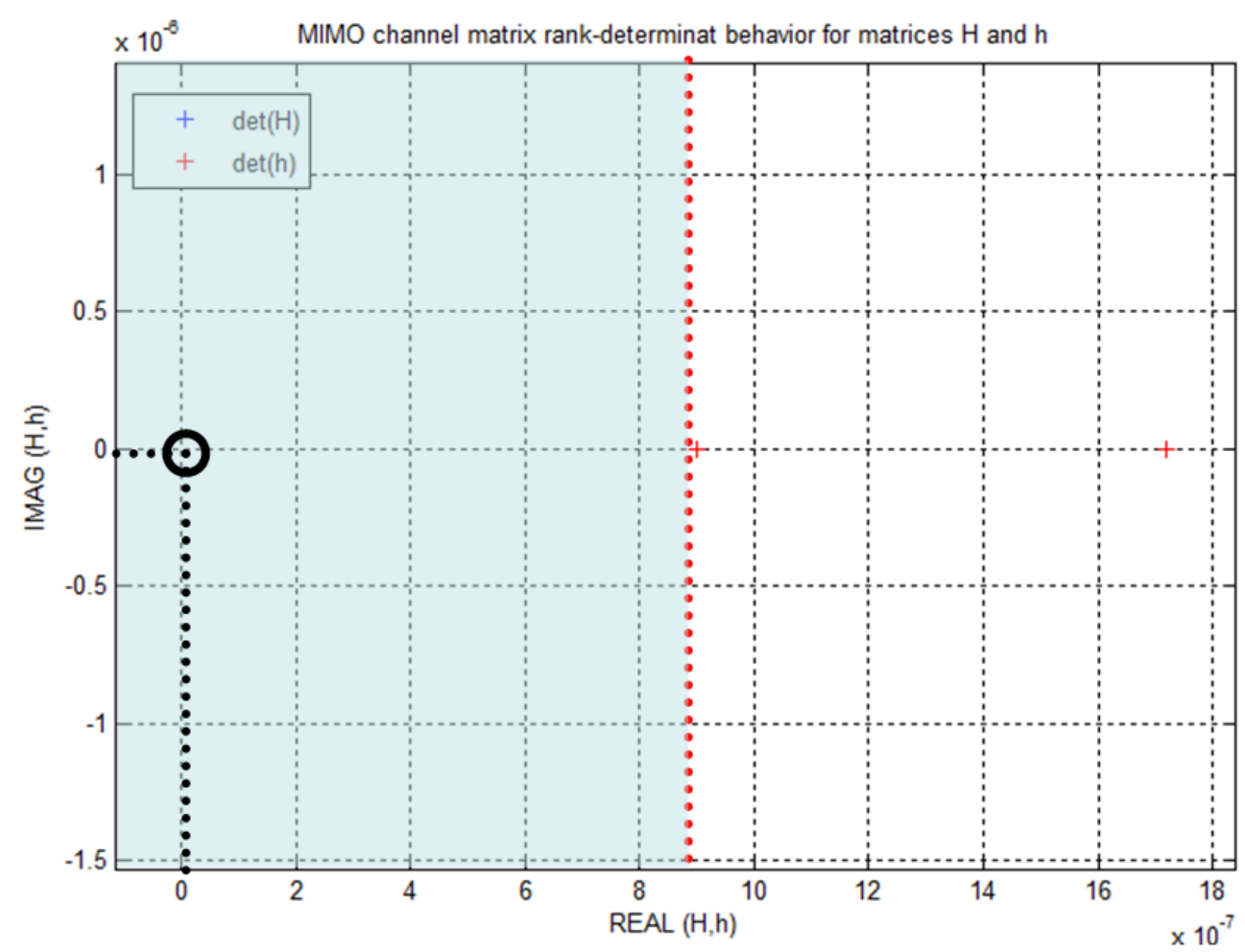

Figure 5. MIMO channel matrix rank-determinant behavior for several realizations forh. Full-rank properties for hand $h^{\top} h$ preveal for RFC-based environments (light-blue region delimited by red-dotted line).

\section{Proposed algorithm}

The proposal for a novel algorithm for computing a LPI matrix $\mathrm{h}^{+} \in \mathbb{R}^{2 n_{T} \times 2 n_{R}}\left(\right.$ with $n_{R} \geq n_{T}$ ) is based on the block-matrix structure of $h$ as exhibited in (4). This idea is an extension of the approach presented in [22]. The existence for this Generalized-Inverse matrix is supported on the statistical properties of the slow-flat quasi-static RFC scenario which impact directly on the singularity of $H$ at every MIMO channel matrix realization. Keeping in mind that other approaches attempting to solve the block-matrix inversion problem [7-10] requires several constraints and conditions, the subsequent proposal does not require any restriction at all mainly due to the aforementioned properties of $H$. From (4), it is suggested that $\left[\begin{array}{l}x^{\mathrm{r}} \\ x^{\mathrm{i}}\end{array}\right]$ is somehow related to $\left[\begin{array}{cc}\mathfrak{R}\left\{H^{+}\right\} & -\mathfrak{J}\left\{H^{+}\right\} \\ \mathfrak{J}\left\{H^{+}\right\} & \mathfrak{R}\left\{H^{+}\right\}\end{array}\right] \cdot \mathrm{Y}$; hence, calculating $\mathrm{h}^{+}$will lead to this solution. 
Let $A=H^{\mathrm{r}}$ and $B=H^{\mathrm{i}}$. It is kwon a priori that $\rho(A+j B)=n_{T}$. Then $\mathrm{h}=\left[\begin{array}{cc}A & -B \\ B & A\end{array}\right]$ with $\rho(\mathrm{h})=2 n_{T}=N_{t}$. Define the matrix $\widetilde{\Omega}$ as $\widetilde{\Omega} \doteq \mathrm{h}^{\mathrm{T}} \mathrm{h} \in \mathbb{R}^{N_{t} \times N_{t}}$, where $\widetilde{\Omega}=\left[\begin{array}{cc}M & -L \\ L & M\end{array}\right]$ with $M=A^{\mathrm{T}} A+B^{\mathrm{T}} B \in \mathbb{R}^{n_{T} \times n_{T}}, L=A^{\mathrm{T}} B-\left(A^{\mathrm{T}} B\right)^{\mathrm{T}} \in \mathbb{R}^{n_{T} \times n_{T}}$, and $\rho(\widetilde{\Omega})=N_{t}$ as a direct consequence from $2 n_{R} \geq 2 n_{T} \rightarrow N_{r} \geq N_{t}$. It can be seen that

$$
\mathrm{h}^{+}=\widetilde{\Omega}^{-1} \mathrm{~h}^{\mathrm{T}} \in \mathbb{R}^{N_{t} \times N_{r}}
$$

For simplicity, matrix operations involved in (12) require classic multiply-and-accumulate operations between row-entries of $\widetilde{\Omega}^{-1} \in \mathbb{R}^{N_{t} \times N_{t}}$ and column-entries ofh ${ }^{\mathrm{T}} \in \mathbb{R}^{N_{t} \times N_{r}}$. Notice immediately that the critical and essential task of computing $\mathrm{h}^{+}$relies on finding the block matrix inverse $\widetilde{\Omega}^{-15}$. The strategy to be followed in order to solve $\widetilde{\Omega}^{-1}$ in (12) will consist of the following steps: 1 ) the proposition of partitioning $\widetilde{\Omega}$ without any restriction on rank-defficiency over inner matrix sub-blocks; 2 ) the definition of iterative multiply-and-accumulate operations within sub-blocks comprised in $\widetilde{\Omega} ; 3$ ) the recursive definition for compacting the overall blockwise matrix inversion. Keep in mind that matrix $\widetilde{\Omega}$ can be also viewed as $\widetilde{\Omega}=\left[\begin{array}{ccc}\widetilde{\omega}_{1,1} & \cdots & \widetilde{\omega}_{1, N_{t}} \\ \vdots & \ddots & \vdots \\ \widetilde{\omega}_{N_{t},} & \cdots & \widetilde{\omega}_{N_{t}, N_{t}}\end{array}\right]$. The symmetry presented in $\widetilde{\Omega}=\left[\begin{array}{cc}M & -L \\ L & M\end{array}\right]$ will motivate the development for the pertinent LPI-based algorithm. From (12) and by the use of Lemma 1 it can be concluded that $\widetilde{\Omega}^{-1}=\left[\begin{array}{cc}Q & P \\ -P & Q\end{array}\right], \quad$ where $Q=\left(M+L M^{-1} L\right)^{-1} \in \mathbb{R}^{n_{T} \times n_{T}}$, $P=Q X \in \mathbb{R}^{n_{T} \times n_{T}}$, and $X=L M^{-1} \in \mathbb{R}^{n_{T} \times n_{T}}$. Interesting enough, full-rank is identified at each matrix sub-block in the main diagonal of $\widetilde{\Omega}$ (besides $\rho(Q)=n_{T}$ ). This structural behavior serves as the leitmotiv for the construction of an algorithm for computing the blockwise inverse $\widetilde{\Omega}^{-1}$. Basically speaking and concerning step 1) of this strategy, the matrix partition procedure obeys the assignments (13-16) defined as:

$$
\begin{gathered}
W_{k}=\left[\begin{array}{cc}
\widetilde{\omega}_{N_{t}-(2 k+1), N_{t}-(2 k+1)} & \widetilde{\omega}_{N_{t}-(2 k+1), N_{t}-2 k} \\
\widetilde{\omega}_{N_{t}-2 k, N_{t}-(2 k+1)} & \widetilde{\omega}_{N_{t}-2 k, N_{t}-2 k}
\end{array}\right] \in \mathbb{R}^{2 \times 2} \\
X_{k}=\left[\begin{array}{ccc}
\widetilde{\omega}_{N_{t}-(2 k+1), N_{t}-(2 k-1)} & \ldots & \widetilde{\omega}_{N_{t}-(2 k+1), N_{t}} \\
\widetilde{\omega}_{N_{t}-2 k, N_{t}-(2 k-1)} & \ldots & \widetilde{\omega}_{N_{t}-2 k, N_{t}}
\end{array}\right] \in \mathbb{R}^{2 \times 2 k}
\end{gathered}
$$

5 Notice that . Moreover, , where and . 


$$
\begin{gathered}
Y_{k}=\left[\begin{array}{cc}
\widetilde{\omega}_{N_{t}-(2 k-1), N_{t}-(2 k+1)} & \widetilde{\omega}_{N_{t}-(2 k-1), N_{t}-2 k} \\
\vdots & \vdots \\
\widetilde{\omega}_{N_{t}, N_{t}-(2 k+1)} & \widetilde{\omega}_{N_{t}, N_{t}-2 k}
\end{array}\right] \in \mathbb{R}^{2 k \times 2} \\
Z_{0}=\left[\begin{array}{cc}
\widetilde{\omega}_{N_{t}-1, N_{t}-1} & \widetilde{\omega}_{N_{T}-1, N_{t}} \\
\widetilde{\omega}_{N_{t}, N_{t}-1} & \widetilde{\omega}_{N_{t}, N_{t}}
\end{array}\right] \in \mathbb{R}^{2 \times 2}
\end{gathered}
$$

The matrix partition over $\widetilde{\Omega}$ obeys the indexk $=1: 1:\left(N_{t} / 2-1\right)$. Because of the even-rectangular dimensions of $\widetilde{\Omega}$, matirx $\widetilde{\Omega}$ owns exactly an amount of $N_{t} / 2=n_{T}$ sub-block matrices of $2 \times 2$ dimension along its main diagonal. Interesting enough, due to RFC-based environment characteristics studied in (1) and (4), it is found that:

$$
\rho\left(W_{k}\right)=\rho\left(Z_{0}\right)=2
$$

After performing these structural characteristics for $\widetilde{\Omega}$, and with the use of (13-16), step 2) of the strategy consists of the following iterative operations also indexed by $k=1: 1:\left(N_{t} / 2-1\right)$, in the sense of performing:

$$
\begin{gathered}
\varphi_{k}=W_{k}-X_{k} Z_{k-1}^{-1} Y_{k} \\
\alpha_{k}=\varphi_{k}^{-1} X_{k} Z_{k-1}^{-1} \\
\theta_{k}=Z_{k-1}^{-1}+Z_{k-1}^{-1} Y_{k} \alpha_{k}
\end{gathered}
$$

Here: $Z_{k-1}^{-1} \in \mathbb{R}^{2 k \times 2 k}, \varphi_{k} \in \mathbb{R}^{2 \times 2}, \alpha_{k} \in \mathbb{R}^{2 \times 2 k}$, and $\theta_{k} \in \mathbb{R}^{2 k \times 2 k}$. Steps stated in (18-20) help to construct intermediate sub-blocks as

$\widetilde{\Omega}_{k}$ 
yielding correspondingly $W_{k}^{-1}, Z_{0}^{-1}$, and $\varphi_{k}^{-1}$ ) are required to be performed throughout this iterative-recursive process, unlike the operation linked to $Z_{k-1}^{-1}$, which comes from a previous updating step associated with the recursion belonging to $Z_{k}^{-1}$. Although $\rho(\widetilde{\Omega})=N_{t}$ assures the existance of $\widetilde{\Omega}^{-1}$, full-rank requirements outlined in (17) and non-zero determinants for (18) are strongly needed for this iterative-recursive algorithm to work accordingly. Also, full-rank is expected for every recursive outcome related to $Z_{k}^{-1}\left(Z_{k-1}^{-1}\right)$. Again, thank to the characteristics of the slow-flat quasi-static RFC-based environment in which these operations are involved among every MIMO channel matrix realization, conditions in (17) and full-rank of (18) are always satisfied. These issues are corroborated with the aid of the same MATLAB-based simulation framework used to validate full-rank properties over $H$ andh. The statistical evolution for the determinants for $W_{k}, Z_{0}$, and $\varphi_{k}$, and the behavior of singularity within the $Z_{k}^{-1}\left(Z_{k-1}^{-1}\right)$ recursion are respectively illustrated in figures (6)-(8). $\operatorname{MIMO}(2,2), \operatorname{MIMO}(4,2)$, and $\operatorname{MIMO}(4,4)$ were the MIMO communication link configurations considered for these tests. These simulation-driven outcomes provide supportive evidence for the proper functionality of the proposed iterative-recursive algorithm for computing $\widetilde{\Omega}^{-1}$ involving matrix sub-block inversions. On each figure, the statistical evolution for the determinants associated with $Z_{0}, W_{k}, \varphi_{k}$, and $Z_{k}^{-1}\left(Z_{k-1}^{-1}\right)$ are respectively indicated by labels $\operatorname{det}(\mathrm{Zo}), \operatorname{det}(\mathrm{Wk}), \operatorname{det}(\mathrm{Fik})$, and $\operatorname{det}(\mathrm{iZk}, \mathrm{iZkm1})$, while the light-blue zone at bottom delimited by a red-dotted line exhibits the gap which marks the avoidance in rank-deficincy over the involved matrices. The zero-determinant value is marked with a black circle.

The next point of analysis for the behavior of the $\mathrm{h}^{+}$LPI-based iterative-recursive algorithm is complexity, which in essence will consist of a demand in matrix partitions (amount of matrix sub-blocks: PART) and arithmetic operations (amount of additions-subtractions: ADDSUB; multiplications: MULT; and divisions: DIV). Let PART-mtx and ARITH-ops be the nomenclature for complexity cost related to matrix partitions and arithmetic operations, respectively. Without loss of generalization, define $C[*]$ as the complexity in terms of the costs PART-mtx and ARITH-ops belonging to operations involved in $*$. Henceforth, $C\left[\mathrm{~h}^{+}\right]=C\left[\widetilde{\Omega}^{-1}\right]+C\left[\widetilde{\Omega}^{-1} \cdot \mathrm{h}^{\mathrm{T}}\right]$ denotes the cost of computing $\mathrm{h}^{+}$as the sum of the costs of inverting $\widetilde{\Omega}$ and multiplying $\widetilde{\Omega}^{-1}$ byh $^{\mathrm{T}}$. It is evident that: a) $C\left[\widetilde{\Omega}^{-1} \cdot \mathrm{h}^{\mathrm{T}}\right]$ implies PART $=0$ and ARITH-ops itemized into MULT $=8 n_{R} n_{T}^{2}, \quad$ ADD-SUB $=4 n_{R} n_{T}\left(2 n_{T}-1\right)$, and DIV=0; b) $C\left[\widetilde{\Omega}^{-1}\right]=C\left[\mathrm{~h}^{\mathrm{T}} \cdot \mathrm{h}\right]+C\left[\left(\mathrm{~h}^{\mathrm{T}} \mathrm{h}\right)^{-1}\right]$. Clearly, $C\left[\mathrm{~h}^{\mathrm{T}} \cdot \mathrm{h}\right]$ demands no partitions at all, but with a ARITH-ops cost of MULT $=8 n_{R} n_{T}^{2}$, and ADD-SUB $=4\left(2 n_{R}-1\right) n_{T}^{2}$. However, the principal complexity relies critically on $C\left[\left(\mathrm{~h}^{\mathrm{T}} \mathrm{h}\right)^{-1}\right]$, which is the backbone forh ${ }^{+}$, as presented in [22]. Table 2 summerizes these complexity results. For this treatment, $C\left[\left(\mathrm{~h}^{\mathrm{T}} \mathrm{h}\right)^{-1}\right]$ consists of $3 n_{T}-2$ partitions, MULT $=\sum_{k=1}^{n_{T}-1} C_{k}^{I}+6, \mathrm{ADD}-\mathrm{SUB}=\sum_{k=1}^{n_{T}-1} C_{k}^{I I}+1$, and DIV $=\sum_{k=1}^{n_{T}-1} C_{k}^{I I I}+1$. The ARITH-ops cost depends on $C_{k}^{I}, C_{k}^{I I}$, and $C_{k}^{I I I}$; the constant factors for each one of these items are proper of the complexity presented in $C\left[Z_{0}^{-1}\right]$. The remain of the complexities, i.e. $C_{k}^{I}, C_{k}^{I I}$, and $C_{k}^{I I I}$, are 
calculated according to the iterative stpes defined in (18-20) and (21), particularly expressed in terms of

$$
C\left[\varphi_{k}^{-1}\right]+C\left[-\alpha_{k}\right]+C\left[-\theta_{k} Y_{k} W_{k}^{-1}\right]+C\left[\theta_{k}\right]
$$

It can be checked out that: a) no PART-mtx cost is required; b) the ARITH-ops cost employs (22) for each item, yielding: $C_{k}^{I}=40 k^{2}+24 k+12$ (for MULT), $C_{k}^{I I}=40 k^{2}+2$ (for ADD_SUB), and $C_{k}^{I I I}=2($ for DIV).

An illustrative application example is given next. It considers a MIMO channel matrix realization obeying statistical behavior according to (1) and a $\mathrm{MIMO}(4,4)$ configuration:

$$
\begin{aligned}
& H=\left[\begin{array}{cccc}
-0.3059+j 0.7543 & -0.8107+j 0.2082 & 0.2314-j 0.4892 & -0.416-j 1.0189 \\
-1.1777+j 0.0419 & 0.8421-j 0.9448 & 0.1235+j 0.6067 & 1.5437+j 0.4039 \\
0.0886-j 0.0676 & 0.8409+j 0.5051 & -0.132+j 0.8867 & -0.0964-j 0.2828 \\
0.2034-j 0.5886 & -0.0266+j 1.148 & 0.5132-j 1.1269 & 0.0806+j 0.4879
\end{array}\right] \in \mathbb{C}^{4 \times 4} \\
& \text { with } \rho(H)=4 \text {. } \\
& \text { As } \\
& \text { a } \\
& \text { consequence, }
\end{aligned}
$$

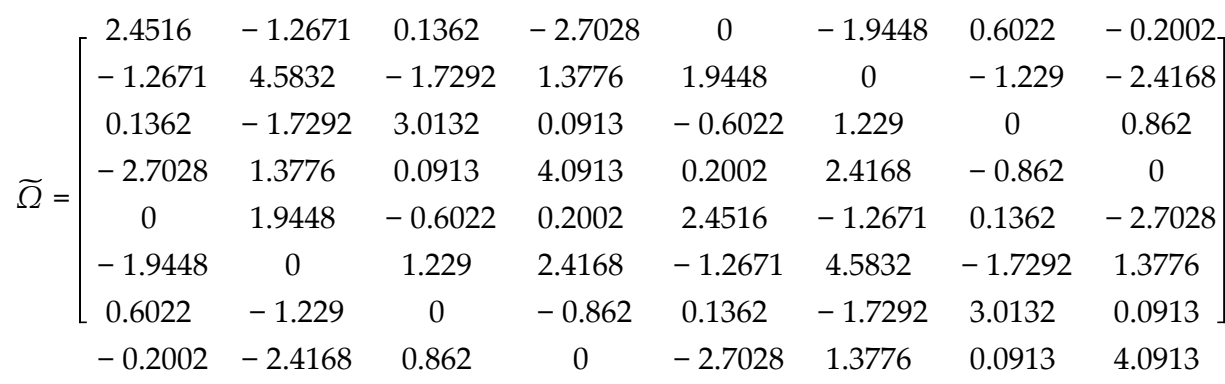

$$
\begin{aligned}
& \in \mathbb{R}^{8 \times 8} \text { with }
\end{aligned}
$$

$$
\rho(\widetilde{\Omega})=8
$$




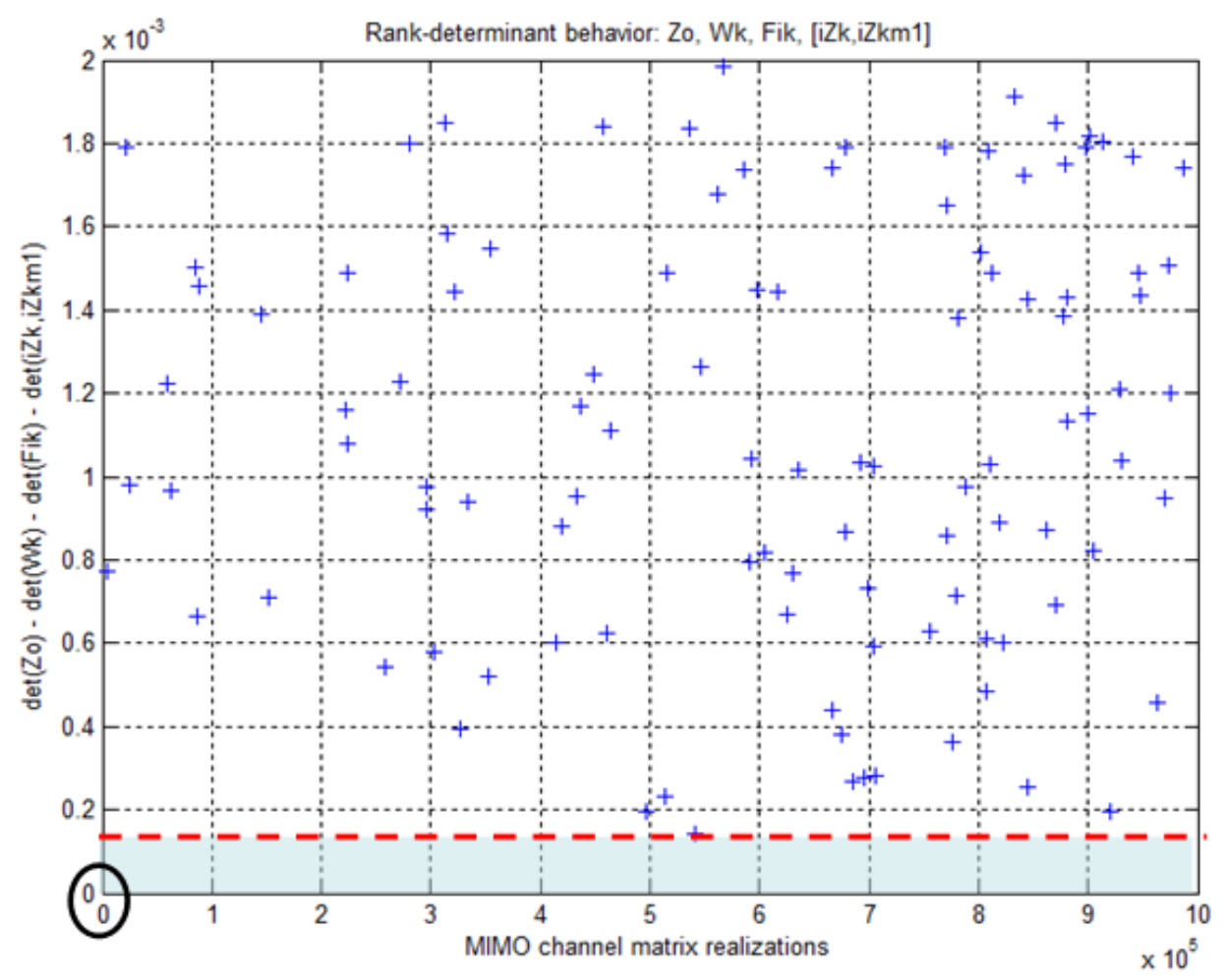

Figure 6. Statistical evolution of the rank-determinant behaviour concerning $Z_{0}, W_{k}, k$, and $Z_{k}^{-1}\left(Z_{k-1}^{-1}\right)$ for a $\mathrm{MIMO}(2,2)$ configuration. 


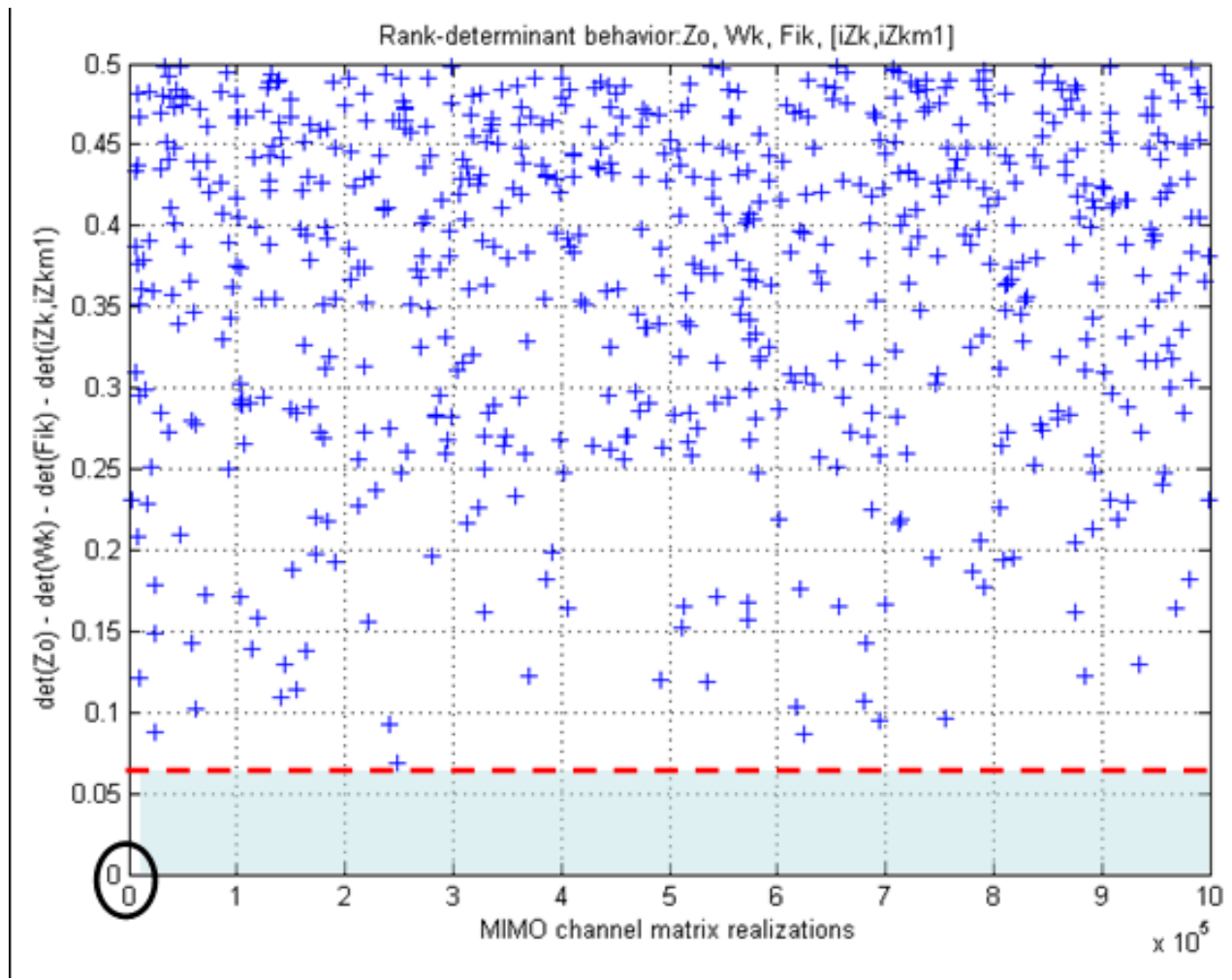

Figure 7. Statistical evolution of the rank-determinant behaviour concerning $Z_{0}, W_{k}, k$, and $Z_{k}^{-1}\left(Z_{k-1}^{-1}\right)$ for a $\mathrm{MIMO}(4,2)$ configuration. 


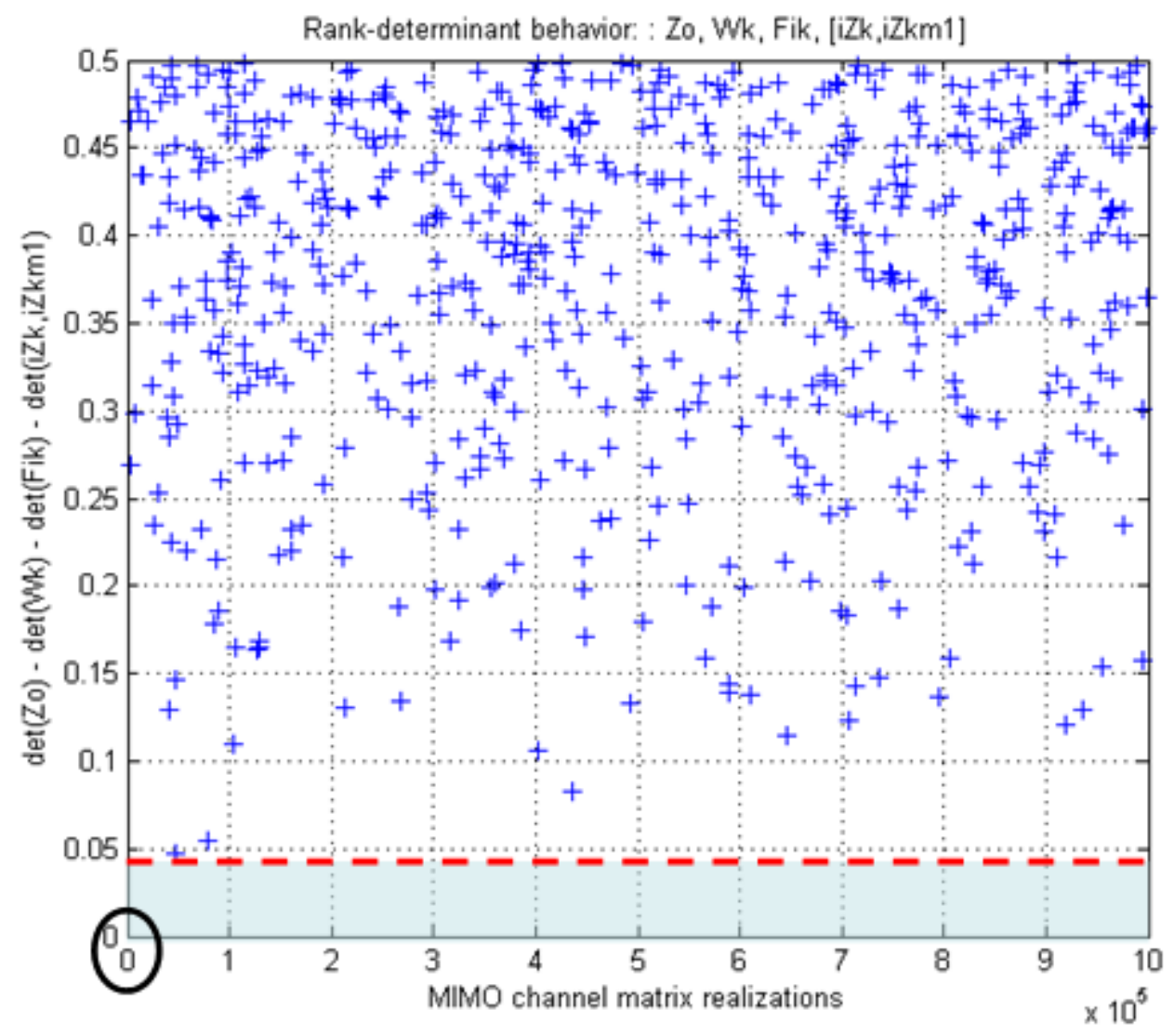

Figure 8. Statistical evolution of the rank-determinant behaviour concerning $Z_{0}, W_{k}, k$, and $Z_{k}^{-1}\left(Z_{k-1}^{-1}\right)$ for a $\mathrm{MIMO}(4,4)$ configuration. 


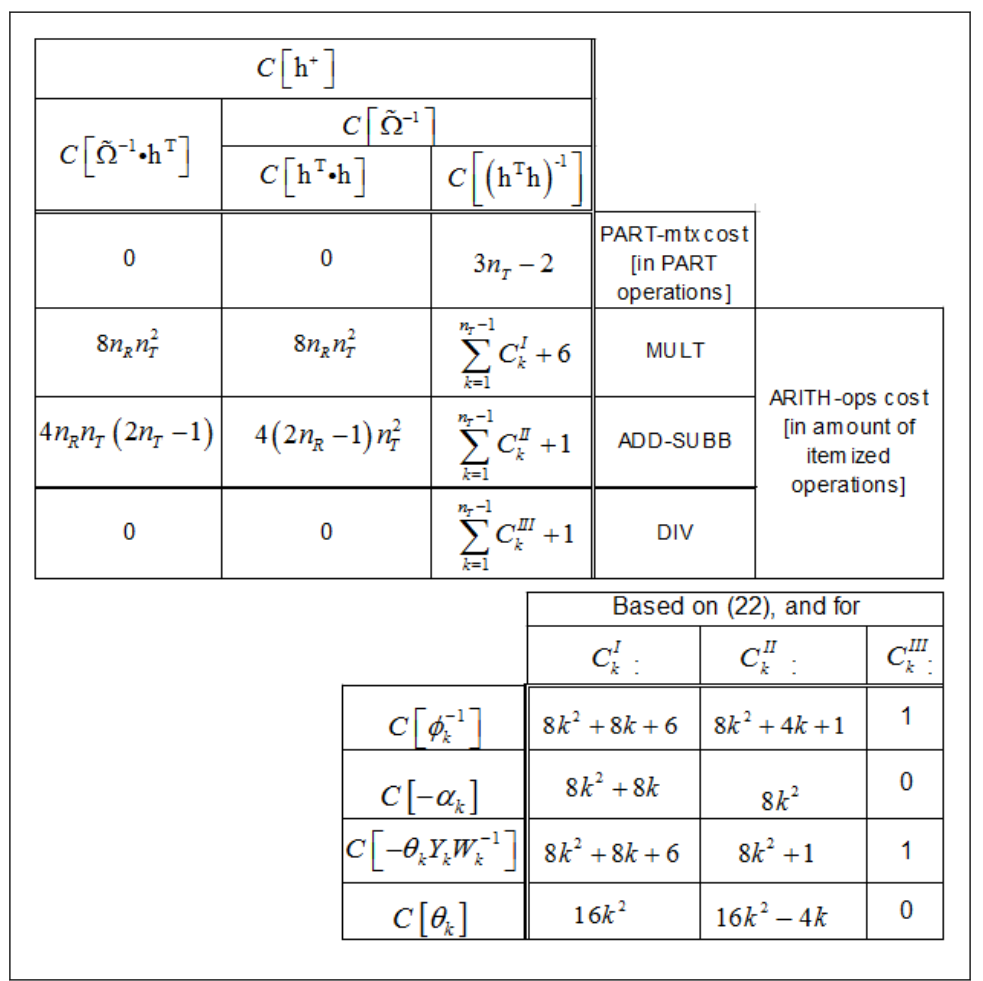

Table 2. Complexity cost results of the LPI-based iterative-recursive algorithm forh ${ }^{+}$.

Applying partition criteria (13-16) and givenk $=1: 1: 3$, the following matrix sub-blocks are generated:

$$
\begin{aligned}
& W_{1}=\left[\begin{array}{cc}
2.4516 & -1.2671 \\
-1.2671 & 4.5832
\end{array}\right], \\
& X_{1}=\left[\begin{array}{cc}
0.1362 & -2.7028 \\
-1.7292 & 1.3776
\end{array}\right], Y_{1}=\left[\begin{array}{cc}
0.1362 & -1.7292 \\
-2.7028 & 1.3776
\end{array}\right], Z_{0}=\left[\begin{array}{ll}
3.0132 & 0.0913 \\
0.0913 & 4.0913
\end{array}\right] \\
& W_{2}=\left[\begin{array}{ll}
3.0132 & 0.0913 \\
0.0913 & 4.0913
\end{array}\right]
\end{aligned}
$$$$
X_{2}=\left[\begin{array}{cccc}
-0.6022 & 1.2290 & 0 & 0.862 \\
0.2002 & 2.4168 & -0.862 & 0
\end{array}\right], Y_{2}=\left[\begin{array}{cc}
-0.6022 & 0.2002 \\
1.229 & 2.4168 \\
0 & -0.862 \\
0.862 & 0
\end{array}\right] \text {, }
$$ 


$$
\begin{aligned}
& W_{3}=\left[\begin{array}{cc}
2.4516 & -1.2671 \\
-1.2671 & 4.5832
\end{array}\right], \\
& X_{3}=\left[\begin{array}{cccccc}
0.1362 & -2.7028 & 0 & -1.9448 & 0.6022 & -0.2002 \\
-1.7292 & 1.3776 & 1.9448 & 0 & -1.229 & -2.4168
\end{array}\right],
\end{aligned}
$$

and $Y_{3}=\left[\begin{array}{cc}0.1362 & -1.7292 \\ -2.7028 & 1.3776 \\ 0 & 1.9448 \\ -1.9448 & 0 \\ 0.6022 & -1.229 \\ -0.2002 & -2.4168\end{array}\right.$ ed as:

$$
\begin{array}{ll}
\varphi_{1}=W_{1}-X_{1} Z_{0}^{-1} Y_{1}, & \alpha_{1}=\varphi_{1}^{-1} X_{1} Z_{0}^{-1}, \theta_{1}=Z_{0}^{-1}+Z_{0}^{-1} Y_{1} \alpha_{1} \\
\varphi_{2}=W_{2}-X_{2} Z_{1}^{-1} Y_{2}, & \alpha_{2}=\varphi_{2}^{-1} X_{2} Z_{1}^{-1}, \theta_{2}=Z_{1}^{-1}+Z_{1}^{-1} Y_{2} \alpha_{2} \\
\varphi_{3}=W_{3}-X_{3} Z_{2}^{-1} Y_{3}, \alpha_{3}=\varphi_{3}^{-1} X_{3} Z_{2}^{-1}, \theta_{3}=Z_{2}^{-1}+Z_{2}^{-1} Y_{3} \alpha_{3}
\end{array}
$$

From (21), the matrix assignments related to recursion $Z_{k}^{-1}\left(Z_{k-1}^{-1}\right)$ produces the following intermediate blockwise matrix results:

$$
\begin{aligned}
& Z_{1}^{-1}\left(Z_{0}^{-1}\right)=\widetilde{\Omega}_{1}^{-1}=\left[\begin{array}{cc}
\varphi_{1}^{-1} & -\alpha_{1} \\
-\theta_{1} Y_{1} W_{1}^{-1} & \theta_{1}
\end{array}\right]=\left[\begin{array}{cccc}
1.5765 & 0.1235 & -0.0307 & 1.0005 \\
0.1235 & 0.3332 & 0.1867 & -0.0348 \\
-0.0307 & 0.1867 & 0.4432 & -0.093 \\
1.0005 & -0.0348 & -0.093 & 0.9191
\end{array}\right] \\
& Z_{2}^{-1}\left(Z_{1}^{-1}\right)=\widetilde{\Omega}_{2}^{-1}=\left[\begin{array}{cc}
\varphi_{2}^{-1} & -\alpha_{2} \\
-\theta_{2} Y_{2} W_{2}^{-1} & \theta_{2}
\end{array}\right] \\
& =\left[\begin{array}{cccccc}
0.4098 & 0.0879 & -0.0829 & -0.1839 & -0.0743 & -0.0775 \\
0.0879 & 0.4355 & -0.2847 & -0.3182 & -0.0422 & -0.0985 \\
-0.0829 & -0.2847 & 1.7642 & 0.3393 & 0.0012 & 1.0686 \\
-0.1839 & -0.3182 & 0.3393 & 0.6023 & 0.2376 & 0.0548 \\
-0.0743 & -0.0422 & 0.0012 & 0.2376 & 0.4583 & -0.0738 \\
-0.0775 & -0.0985 & 1.0686 & 0.0548 & -0.0738 & 0.9499
\end{array}\right], \\
& Z_{3}^{-1}\left(Z_{2}^{-1}\right)=\widetilde{\Omega}^{-1}=\left[\begin{array}{cc}
\varphi_{3}^{-1} & -\alpha_{3} \\
-\theta_{3} Y_{3} W_{3}^{-1} & \theta_{3}
\end{array}\right]
\end{aligned}
$$




$=\left[\begin{array}{cccccccc}1.9798 & 0.3808 & -0.1114 & 1.0224 & 0 & 0.3605 & 0.2524 & 0.2183 \\ 0.3808 & 0.6759 & 0.2619 & 0.0856 & -0.3605 & 0 & 0.2368 & 0.1193 \\ -0.1114 & 0.2619 & 0.5493 & -0.0218 & -0.2524 & -0.2368 & 0 & -0.0535 \\ 1.02224 & 0.0856 & -0.0218 & 0.9839 & -0.2183 & -0.1193 & 0.0535 & 0 \\ 0 & -0.3605 & -0.2524 & -0.2183 & 1.9798 & 0.3808 & -0.1114 & 1.0224 \\ 0.3605 & 0 & -0.2368 & -0.1193 & 0.3808 & 0.6759 & 0.2619 & 0.0856 \\ 0.2524 & 0.2368 & 0 & 0.0535 & -0.1114 & 0.2619 & 0.5493 & -0.0218 \\ 0.2183 & 0.1193 & -0.0535 & 0 & -0.1114 & 0.0856 & -0.0218 & 0.9839\end{array}\right]$. This

last recursive outcome from $Z_{k}^{-1}\left(Z_{k-1}^{-1}\right)$ corresponds to $\widetilde{\Omega}^{-1}$, and is further used for calculating $\mathrm{h}^{+}=\widetilde{\Omega}^{-1} \mathrm{~h}^{\mathrm{T}} \in \mathbb{R}^{8 \times 8}$. Moreover, notice that full-rank properties are always presented in matri$\operatorname{ces} Z_{0}, W_{1}, W_{2}, W_{3}, \varphi_{1}, \varphi_{2}, \varphi_{3}, Z_{1}^{-1}, Z_{2}^{-1}$, and $Z_{3}^{-1}$.

\section{VLSI implementation aspects}

The arithmetic operations presented in the algorithm for computing $\mathrm{h}^{+}$can be implemented under a modular-iterative fashion towards a VLSI (Very Large Scale of Integration) design. The partition strategy comprised in (13-16) provides modularity, while (18-20) is naturally associated with iterativeness; recursion is just used for constructing matrix-blocks in (21). Several well-studied aspects aid to implement a further VLSI architecture [23-27] given the nature of the mathematical structure of the algorithm. For instance, systolic arrays [25-27] are a suitable choice for efficient, parallel-processing architectures concerning matrix multiplications-additions. Bidimensional processing arrays are typical architectural outcomes, whose design consist basically in interconnecting processing elements (PE) among different array layers. The configuration of each PE comes from projection or linear mapping techniques [25-27] derived from multiplications and additions presented in (18-20). Also, systolic arrays tend to concurrently perform arithmetic operations dealing with the matrix concatenated multiplications $X_{k} Z_{k-1}^{-1} Y_{k}, \varphi_{k}^{-1} X_{k} Z_{k-1}^{-1}, Z_{k-1}^{-1} Y_{k} \alpha_{k}$, and $\theta_{k} Y_{k} W_{k}^{-1}$ presented in (18-20). Consecutive additions inside every PE can be favourably implemented via Carry-Save-Adder (CSA) architectures [23-24], while multiplications may recur to Booth multipliers [23-24] in order to reduce latencies caused by adding acummulated partial products. Divisions presented in $W_{k}^{-1}, Z_{0}^{-1}$, and $\varphi_{k}^{-1}$ can be built through regular shift-and-subtract modules or classic serial-parallel subtractors [23-24]; in fact, CORDIC (Coordinate Rotate Digital Computer) processors [23] are also employed and configured in order to solve numerical divisions. The aforementioned architectural aspects offer an attractive and alternative framework for consolidating an ultimate VLSI design for implementing the $\mathrm{h}^{+}$algorithm without compromising the overall system data throughput (intrinsicly related to operation frequencies) for it. 


\section{Conclusions}

This chapter presented the development of a novel iterative-recursive algorithm for computing a Left-Pseudoinverse (LPI) as a Generalized-Inverse for a MIMO channel matrix within a Rayleigh fading channel (RFC). The formulation of this algorithm consisted in the following step: i) first, structural properties for the MIMO channel matrix acquired permanent fullrank due to statistical properties of the RFC scenario; ii) second, Partition-Matrix Theory was applied allowing the generation of a block-matrix version of the MIMO channel matrix; iii) third, iterative addition-multiplication operations were applied at these matrix subblocks in order to construct blockwise sub-matrix inverses, and recursively reusing them for obtaining the LPI. For accomplishing this purpose, required mathematical background and MIMO systems concepts were provided for consolidating a solid scientific framework to understand the context of the problem this algorithm was attempting to solve. Proper functionality for this approach was validated through simulation-driven experiments, as well as providing an example of this operation. As an additional remark, some VLSI aspects and architectures were outlined for basically implementing arithmetic operations within the proposed LPI-based algorithm.

\section{Acknowledgement}

This work was supported by CONACYT (National Council of Science and Technology) under the supervision, revision, and sponsorship of ITESM University (Instituto Tecnológico y de Estudios Superiores de Monterrey).

\section{Author details}

P. Cervantes ${ }^{1}$, L. F. González ${ }^{1}$, F. J. Ortiz ${ }^{2}$ and A. D. García ${ }^{2}$

Instituto Tecnológico y de Estudios Superiores de Monterrey, Campus Guadalajara, ITESM University,, Mexico

Instituto Tecnológico y de Estudios Superiores de Monterrey, Campus Estado de México, ITESM University,, Mexico

\section{References}

[1] Abramovich YI, Johnson BA, and Spencer NK(2008). Two-Dimensional Multivariate Parametric Models for Radar Applications-Part I: Maximum-Entropy Extensions for Toeplitz-Block Matrices. IEEE Transactions on Signal Processing, November 2008: 5509-5526., 56(11) 
[2] Bera TK, et al(2011). Improving the image reconstruction in Electrical Impedance Tomography (EIT) with block matrix-based Multiple Regularization (BMMR): A practical phantom study. World Congress on Information and Communication Technologies (WICT). , 2011, 1346-1351.

[3] Kailath, T. (1980). Linear Systems. Prentice-Hall. 682 p.

[4] Spong MW(1998). Underactuated Mechanical Systems. Control Problems in Robotics and Automation, Lecture Notes in Control and Information Sciences, Springer-Verlag: (230), 135-150.

[5] Utkin, V., Guldner, J., \& Shi, J. X. (1992). Sliding Mode Control in Electro-Mechanical Systems. CRC Press. April 1999: 338 p.

[6] Juang J-N(1993). Applied System Identification. Prentice Hall. 400 p.

[7] Watt SM(2006). Pivot-Free Block Matrix Inversion. Proceedings of the Eighth International Symposium on Symbolic and Numeric Algorithms for Scientific Computing (SYNASC), IEEE Computer Society: 5 p.

[8] Tian, Y., \& Tanake, Y. (2009). The inverse of any two-by-two nonsingular partitioned matrix and three matrix inverse completion problems. Journal Computers \& Mathematics with Applications, April 2009: 12 p., 57(8)

[9] Choi, Y. (2009). New Form of Block Matrix Inversion. International Conference on Advanced Intelligent Mechatronics. July , 2009, 1952-1957.

[10] Choi, Y., \& Cheong, J. (2009). New Expressions of 2 X2 Block Matrix Inversion and Their Application. IEEE Transactions on Automatic Control, November 2009: 2648-2653., 54(11)

[11] Fontán FP, and Espiñera PM(2008). Modeling the Wireless Propagation Channel. Wiley. $268 \mathrm{p}$.

[12] El -Hajjar, M., \& Hanzo, L. (2010). Multifunctional MIMO Systems: A Combined Diversity and Multiplexing Design Perspective. IEEE Wireless Communications. April, 2010, 73-79.

[13] Biglieri, E., et al. (2007). MIMO Wireless Communications. Cambridge University Press: United Kingdom. 344 p.

[14] Jankiraman, M. (2004). Space-Time Codes and MIMO Systems. Artech House: United States. 327 p.

[15] Biglieri, E., Proakis, J., \& Shamai, S. (1998). Fading Channels: Information-Theoretic and Communications Aspects. IEEE Transactions on Information Theory, October 1998: 2619-2692., 44(6)

[16] Almers, P., Bonek, E., Burr, A., et al. (2007). Survey of Channel and Radio Propagation Models for Wireless MIMO Systems. EURASIP Journal on Wireless Communications and Networking, January 2007: 19 p., 2011(1) 
[17] Golub GH, and Van Loan CF(1996). Matrix Computations. The Johns Hopkins University Press. 694 p.

[18] Serre, D. (2001). Matrices: Theory and Applications. Springer Verlag. 202 p.

[19] R\&S®. Rohde \& Schwarz GmbH \& Co. KG. WLAN 802.11n: From SISO to MIMO. Application Note: 1MA179_9E. Available: www.rohde-schwarz.com:p.

[20] Agilent, @ ○., \& Technologies, Inc. (2008). Agilent MIMO Wireless LAN PHY Layer [RF] : Operation \& Measurement: Application Note: 1509. Available: www.agilent.com:p.

[21] Paul, T., \& Ogunfunmi, T. (2008). Wireless LAN Comes of Age : Understanding the IEEE 802.11n Amendment. IEEE Circuits and Systems Magazine. First Quarter , 2008, 28-54.

[22] Cervantes, P., González, V. M., \& Mejía, P. A. (2009). Left-Pseudoinverse MIMO Channel Matrix Computation. 19th International Conference on Electronics, Communications, and Computers (CONIELECOMP 2009). July , 2009, 134-138.

[23] Milos, E., \& Tomas, L. (2004). Digital Arithmetic. Morgan Kauffmann Publishers. 709 p.

[24] Parhi KK(1999). VLSI Digital Signal Processing Systems: Design and Implementation. John Wiley \& Sons. 784 p.

[25] Song SW(1994). Systolic Algorithms: Concepts, Synthesis, and Evolution. Institute of Mathematics, University of Sao Paulo, Brazil. Available: http://www.ime.usp.br/ song/papers/cimpa.pdf. DOIp.(10)

[26] Kung SY(1985). VLSI Array Processors. IEEE ASSP Magazine. July , 1985, 4-22.

[27] Jagadish, H. V., Rao, S. K., \& Kailath, T. (1987). Array Architectures for Iterative Algorithms. Proceedings of the IEEE, September 1987: 1304-1321., 75(9) 